Research Article

\title{
Modal and Natural Frequency Sensitivity Analysis of Electrohydraulic Stewart Platform
}

\author{
Lijie Zhang, ${ }^{1}$ Xuemei Zong, ${ }^{1,2}$ Yue Tang, ${ }^{1}$ Xinchun Chen, ${ }^{1,2}$ Junxue Feng, \\ and Xiaoming Yuan (iD) 1,2 \\ ${ }^{1}$ Hebei Provincial Key Laboratory of Heavy Machinery Fluid Power Transmission and Control, Yanshan University, \\ Qinhuangdao 066004, China \\ ${ }^{2}$ Jiangsu XCMG Construction Machinery Research Institute Co., Ltd., Xuzhou 221004, China \\ ${ }^{3}$ Beijing Research Institute of Automation for Machinery Industry Co., Ltd., Beijing 100000, China
}

Correspondence should be addressed to Xiaoming Yuan; yuanxiaoming@ysu.edu.cn

Received 23 February 2021; Revised 21 March 2021; Accepted 28 March 2021; Published 7 April 2021

Academic Editor: Ling Zhou; lingzhou@ujs.edu.cn

Copyright $\odot 2021$ Lijie Zhang et al. This is an open access article distributed under the Creative Commons Attribution License, which permits unrestricted use, distribution, and reproduction in any medium, provided the original work is properly cited.

Electrohydraulic Stewart platform is a multi-input and multi-output mechanical-hydraulic coupling system, which has the advantages of large power-to-weight ratio and high accuracy. It has been widely used in construction machinery, aerospace, and other fields. In the actual working process, especially in the high-speed motion, the Stewart platform movement process will produce a large impact and vibration and then affect the stability, accuracy, and service life of the platform. When the frequency of the external excitation coincides with the natural frequency of the electrohydraulic Stewart platform, it may cause the failure of the platform. Therefore, based on the relationship between the volumetric elastic modulus of the gas-bearing oil and the hydraulic stiffness of the leg, a mechanical-hydraulic coupling dynamic model of the electrohydraulic Stewart platform was established, and the natural frequencies and modal shapes of the platform were analyzed under typical conditions. The sensitivity calculation formula of the natural frequency of the system on the upper platform mass and the hydraulic stiffness of the outer leg is given by an analytical method, and the influence law of the upper platform mass and the outer leg stiffness on the natural frequency and the sensitivity of the electrohydraulic Stewart platform under typical conditions is discussed. This study can provide theoretical support for dynamic optimization of the electrohydraulic Stewart platform.

\section{Introduction}

The six-degree-of-freedom platform, also known as the Stewart platform, was designed and proposed by the British engineer Stewart in 1965. It is a typical parallel mechanism, originally used as a flight simulator for pilot training. In the later stage, the electrohydraulic Stewart platform appeared on the basis of combining the hydraulic transmission system. Compared with the series simulator platform, the electrohydraulic Stewart platform has the advantages of large power-to-weight ratio, large stiffness, small inertia, and high precision [1-3] and is currently widely used in various fields such as machine tool [4], medical equipment [5], vehicle $[6,7]$, spacecraft $[8,9]$, and telescope $[10,11]$.The design and development of the electrohydraulic Stewart platform involve multiple disciplinary fields such as machinery [12], fluid transmission and control [13, 14], computer [15], and sensor [16].The electrohydraulic Stewart platform is a multi-input multi-output system with strong coupling because its legs cooperate with each other to achieve the corresponding position and attitude in the design space. When the platform is working, the lower platform is generally fixed, so its dynamic characteristics are mainly affected by the stiffness of the six supporting legs and the mass of the upper platform. Compared with the direct motor drive, the leg stiffness of the electrohydraulic Stewart platform has been improved to a certain extent, but it is still significantly lower than that of the mechanical components. Therefore, the dynamic characteristics of the platform will be significantly different with the change of the structure. When the frequency of external excitation is close to its natural frequency, the vibration and destruction of the platform will 
be caused no matter which structure is adopted $[17,18]$. Therefore, it is of great significance to analyze the inherent characteristics and parameter sensitivity of the platform to improve its dynamic performance $[19,20]$.

In practical work, especially at high speed and high frequency, the vibration of Stewart platform may be caused by defects of platform components or parts, errors during platform assembly, or incoordination between the control system of the platform and the actual movement of the platform [21-23].With the development and progress of science and technology, instruments and equipment in various fields have higher and higher requirements for accuracy, and such vibration has more and more negative effects on the accuracy of equipment [24-26].For the problem of vibration reduction, Wang et al. proposed a novel Stewart isolation platform system based on a bionic X-shaped structure. In the presence of $\mathrm{X}$-shaped structure, beneficial nonlinear stiffness and nonlinear damping characteristics can be obtained, which is conducive to better vibration isolation performance in multiple directions [27]. Cheng et al. studied the dynamic control problem of Gough-Stewart vibration platform with flexible support structure, simplified the Stewart parallel mechanism into a multibody system with elastic base platform by Newton-Euler method, and proposed a PD control law based on position prediction of two platforms. The simulated wind excitation was used for control simulation, and the control effect was evaluated by root mean square index [28]. For the entire Stewart platform, ensuring that the overall stiffness of the platform reaches the expected value can effectively reduce the vibration caused by the platform motion $[29,30]$.

To study the dynamic characteristics of the Stewart platform, it is necessary to establish and solve its dynamic equation, which mainly includes mass matrix, stiffness matrix, and damping matrix [31,32]. The natural frequency, mode shape, and parameter sensitivity can be further solved by the dynamic equation. For Stewart platform, Lagrange method [33], virtual work principle [34], Newton-Euler method [9], and Kane equation [35] can be used to solve its dynamics problem.

In conclusion, the hydraulic stiffness of the leg and the mass of the upper platform have important effects on the dynamic characteristics of the electrohydraulic Stewart platform, but the natural frequency, mode shape, and parameter sensitivity of the platform need to be further studied. Therefore, based on the relationship between the volumetric elastic modulus of gas-bearing oil and the hydraulic stiffness of the leg, the dynamic equation of the electrohydraulic Stewart platform is established to determine the natural frequency and mode shapes of the platform, and the sensitivity of the natural frequency of the platform to the mass of the upper platform and the hydraulic stiffness of the leg is studied by using the analytical method.

\section{Dynamic Modeling of Electrohydraulic Stewart Platform}

2.1. Composition and Pose Description of the Stewart Platform. As shown in Figure 1, the Stewart 6-DOF platform is composed of an upper platform (moving platform), a lower

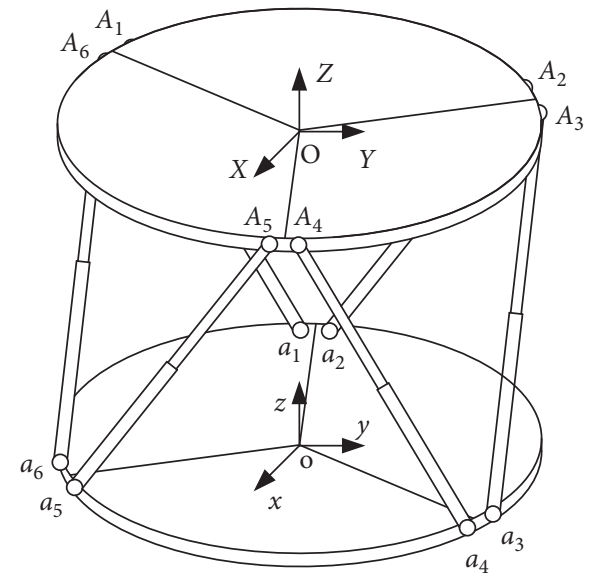

FIgURE 1: Stewart 6-DOF platform geometrical structure diagram.

platform (static platform), and six outer legs with the same structure. The six supporting legs are connected to the moving platform through ball hinge $S$ and to the static platform through hooker hinge $U$. There is a moving pair $P$ on the outer leg, which ensures the movement of the whole platform by exerting driving force on $P$. The mechanism belongs to plane platform type, and the centers of six spherical hinge $S$ and six hooker hinge $U$ are distributed in two planes, respectively.

For the convenience of analysis, the absolute static coordinate system $0-x y z$ is established at the center of mass of the static platform, where $o$ is the center of the circle of the static platform. The relative moving coordinate system $O$ $X Y Z$ is established at the center of mass of the moving platform, where $O$ is the center of the circle of the moving platform. The position of the six spherical hinge $S$ with respect to the relative motion coordinate system $O-X Y Z$ is defined as $A_{1}, A_{2}, \ldots, A_{6}$, and the position of the six Hook hinge $U$ relative to the absolute static coordinate system o-xyz is $a_{1}, a_{2}$, $\ldots, a_{6}$ denotes. The six legs of the platform are $l_{1}, l_{2}, \ldots, l_{6}$ said. The displacement matrix of the moving coordinate system $\mathrm{O}-X Y Z$ relative to the absolute static coordinate system o- $x y z$ is expressed by $\mathbf{E}$, and the rotation matrix is expressed by $\mathbf{R}_{a}$.

The shape and position of the platform can be uniquely determined by six variables $\left(P_{x}, P_{y}, P_{z}, \boldsymbol{\alpha}, \boldsymbol{\beta}\right.$, and $\left.\boldsymbol{\gamma}\right)$ where $P_{x}$, $P_{y}$, and $P_{z}$ represent the projection of the displacement vector of the moving coordinate system $O-X Y Z$ relative to the absolute static coordinate system o- $x y z$ along the axes $x$, $y$, and $z ; \boldsymbol{\alpha}, \boldsymbol{\beta}$, and $\boldsymbol{\gamma}$ are, respectively, the roll angle, pitch angle and yaw angle of rotation of the coordinate system $O$ $X Y Z$ on a moving platform compared with that of the coordinate system $o-x y z$ on a static platform, which are described by $Z Y X$ Euler angle. Then, the displacement matrix $\mathbf{E}$ and rotation matrix $\mathbf{R}_{a}$ can be expressed as

$$
\begin{aligned}
\mathbf{E} & =\left(\begin{array}{lll}
P_{x} & P_{y} & P_{z}
\end{array}\right)^{T}, \\
\mathbf{R}_{\mathbf{a}} & =\left(\begin{array}{ccc}
c \gamma c \boldsymbol{\beta} & c \gamma s \boldsymbol{\beta} \boldsymbol{\beta} \boldsymbol{\alpha}-s \gamma c \boldsymbol{\alpha} & s \gamma s \boldsymbol{\alpha}+c \gamma s \boldsymbol{\beta} c \boldsymbol{\alpha} \\
s \gamma c \boldsymbol{\beta} & c \gamma c \boldsymbol{\alpha}+s \boldsymbol{s} \boldsymbol{\beta} s \boldsymbol{\alpha} & s \gamma s \boldsymbol{\beta} c \boldsymbol{\alpha}-c \gamma c \boldsymbol{\alpha} \\
-s \boldsymbol{\beta} & c \boldsymbol{\beta} s \boldsymbol{\alpha} & c \boldsymbol{\beta} c \boldsymbol{\alpha}
\end{array}\right),
\end{aligned}
$$

where $s \alpha=\sin \alpha$ and $c \alpha=\cos \alpha$. 
The positions of the six supporting legs of the platform can be expressed as

$$
l_{i}=\mathbf{R}_{a} \times \mathbf{A}_{i}+\mathbf{E}-\mathbf{a}_{i},
$$

where $i=1,2, \ldots, 6$.

This paper mainly analyzes the free vibration and sensitivity of the two poses, and the parameters of the two poses are as follows:

Pose 1:

$$
\mathbf{x}=\left(P_{x}, P_{y}, P_{z}, \alpha, \beta, \gamma\right)=\left(\begin{array}{llllll}
0 & 0 & 2 \sqrt{3} & 0 & 0 & 0
\end{array}\right) .
$$

Its graph is shown in Figure 2.

Pose 2:

$$
\mathbf{x}=\left(P_{x}, P_{y}, P_{z}, \alpha, \beta, \gamma\right)=\left(\begin{array}{llllll}
1 & 2 & 2 \sqrt{3} & 10 & 5 & 5
\end{array}\right) .
$$

Its graph is shown in Figure 3.

Among them, pose 1 represents that the electrohydraulic Stewart platform is in the positive position, as shown in Figure 1, and the platform has symmetry. The pose 2 represents the general position of the electrohydraulic Stewart platform; that is, the shape and position parameters of the platform vary and the platform is not symmetrical. Through the analysis of dynamic characteristics of pose 1 and pose 2, the influence of platform position change on its dynamic characteristics was determined.

Physical parameters were obtained by measuring and calculating the existing electrohydraulic Stewart platform in the laboratory, as shown in Table 1.

2.2. Dynamic Equation of the Electrohydraulic Stewart Platform Drive System. The dynamic model of the electrohydraulic Stewart platform adopts the following assumptions:

(1) The components of the Stewart platform are all rigid bodies; that is, the elastic deformation of other components is not considered.

(2) The effect of the six supporting legs on the moving platform is equivalent to a linear spring along the length of the legs.

(3) Since the mass of the upper platform is much larger than the mass of the outer leg, the effect of the outer leg mass on the Stewart platform system is negligible.

(4) The friction generated by relative movement of components is ignored.

(5) Machining and installation errors of all parts are ignored.

2.2.1. Velocity Analysis and Jacobian Matrix. The six variables that determine the shape and position of the platform are set as generalized coordinates:

$$
x=\left(P_{x}, P_{y}, P_{z}, \alpha, \beta, \gamma\right)^{T} .
$$

Then, the velocity $\mathbf{V}$ and angular velocity $\boldsymbol{\omega}$ of the moving platform can be expressed as

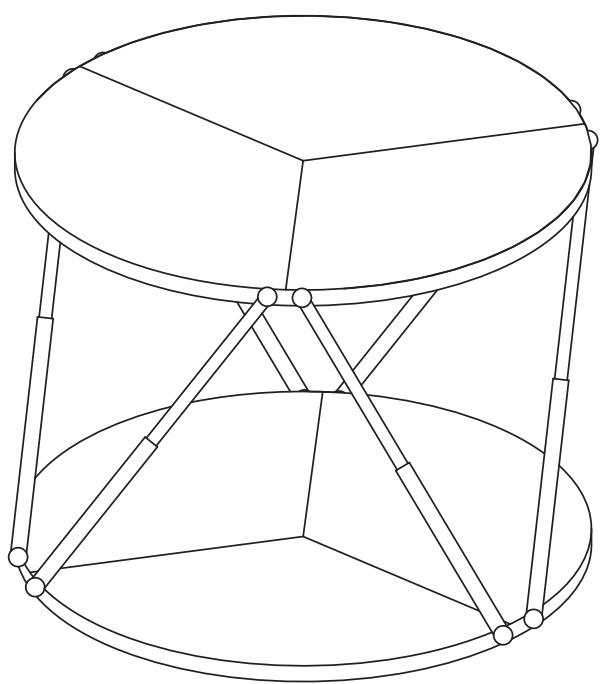

Figure 2: Schematic diagram of 6-DOF platform under pose 1.

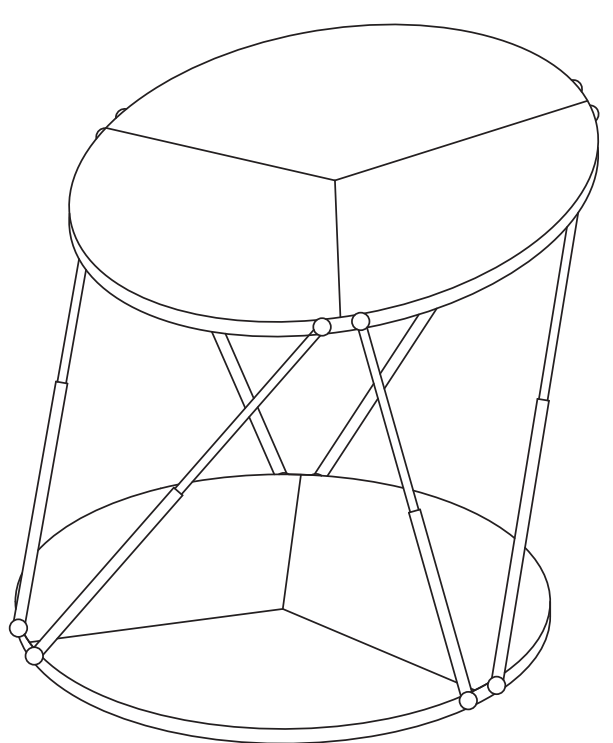

Figure 3: Schematic diagram of 6-DOF platform under pose 2.

$$
\begin{aligned}
& V=\dot{P}=\left(\begin{array}{lll}
\dot{P}_{x} & \dot{P}_{y} & \dot{P}_{z}
\end{array}\right), \\
& \omega=\dot{\theta}=(\dot{\alpha}, \dot{\beta}, \dot{\gamma}) .
\end{aligned}
$$

Let $\mathbf{r}$ be the vector diameter of hinge point $S$ relative to $O$; $\mathbf{V}_{s}$ is the velocity of hinge point $S$; $\mathbf{e}$ is the unit direction vector of bar $l$; and $v$ is the change rate of rod length of $\operatorname{rod} l$, then

$$
\begin{aligned}
r_{i} & =R_{a} A_{i}, \\
V_{a i} & =V+\omega \times r_{i}, \\
v_{i} & =V_{a i} \cdot e_{i},
\end{aligned}
$$

where $i=1,2, \ldots, 6$.

Substituting (8) into (9), we can obtain 
TABLe 1: Physical parameters of electrohydraulic Stewart platform (unit: Si).

\begin{tabular}{|c|c|c|}
\hline Name & Symbol & Value \\
\hline Upper platform radius & $R$ & 2 \\
\hline Thickness of upper platform & $D$ & 0.2 \\
\hline Quality of upper platform & $M$ & 20 \\
\hline Inertia of the upper platform about the $X$-axis & $I_{x}$ & 20.07 \\
\hline Inertia of the upper platform about the $Y$-axis & $I_{y}$ & 20.07 \\
\hline Inertia of upper platform about the $Z$-axis & $I_{z}$ & 40 \\
\hline Hook hinges at position 1 & $\mathbf{a}_{1}$ & {$\left[\begin{array}{llll}(1 / 2) R & \left(\begin{array}{lll}\sqrt{3} & / 2\end{array}\right) R & 0\end{array}\right]_{\mathrm{T}}^{T}$} \\
\hline Hook hinges at position 2 & $\mathbf{a}_{2}$ & {$\left[\begin{array}{llll}(1 / 2) R & (\sqrt{3} & / 2) R & 0\end{array}\right]^{\mathrm{T}}$} \\
\hline Hook hinges at position 3 & $\mathbf{a}_{3}$ & $\left.\left[\begin{array}{lll}(1 / 2) R & -(\sqrt{3} & / 2\end{array}\right) R \quad 0\right]_{T}^{T}$ \\
\hline Hook hinges at position 4 & $\mathbf{a}_{4}$ & $\left.\left[\begin{array}{lll}(1 / 2) R & -(\sqrt{3} & / 2\end{array}\right) R \quad 0\right]^{T}$ \\
\hline Hook hinges at position 5 & $\mathbf{a}_{5}$ & {$\left[\begin{array}{lll}-R & 0 & 0\end{array}\right]^{T}$} \\
\hline Hook hinges at position 6 & $\mathbf{a}_{6}$ & {$\left[\begin{array}{lll}-R & 0 & 0\end{array}\right]^{T}$} \\
\hline Ball hinge at position 1 & $\mathbf{A}_{1}$ & {$\left[\begin{array}{llll}-(1 / 2) R & (\sqrt{3} & / 2) R & 0\end{array}\right]^{T}$} \\
\hline Ball hinge at position 2 & $\mathbf{A}_{2}$ & {$\left[\begin{array}{lll}R & 0 & 0\end{array}\right]^{T}$} \\
\hline Ball hinge at position 3 & $\mathbf{A}_{3}$ & {$\left[\begin{array}{lll}R & 0 & 0\end{array}\right]^{T}$} \\
\hline Ball hinge at position 4 & $\mathbf{A}_{4}$ & {$\left[\begin{array}{lll}-(1 / 2) R & \left(\begin{array}{lll}\sqrt{3} & / 2\end{array}\right) R & 0\end{array}\right]^{T}$} \\
\hline Ball hinge at position 5 & $\mathbf{A}_{5}$ & 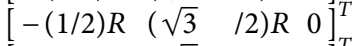 \\
\hline Ball hinge at position 6 & $\mathbf{A}_{6}$ & {$\left[\begin{array}{llll}-(1 / 2) R & (\sqrt{3} & / 2) R & 0\end{array}\right]^{T}$} \\
\hline
\end{tabular}

$$
v_{i}=\left[\begin{array}{ll}
e_{i}^{T} & \left(r_{i} \times e_{i}\right)^{T}
\end{array}\right]\left[\begin{array}{l}
V \\
\omega
\end{array}\right] .
$$

For all six legs, there is

$$
\left[\begin{array}{c}
v_{1} \\
v_{2} \\
v_{3} \\
v_{4} \\
v_{5} \\
v_{6}
\end{array}\right]=\left[\begin{array}{cc}
e_{1}^{T} & \left(r_{1} \times e_{1}\right)^{T} \\
e_{2}^{T} & \left(r_{2} \times e_{2}\right)^{T} \\
e_{3}^{T} & \left(r_{3} \times e_{3}\right)^{T} \\
e_{4}^{T} & \left(r_{4} \times e_{4}\right)^{T} \\
e_{5}^{T} & \left(r_{5} \times e_{5}\right)^{T} \\
e_{6}^{T} & \left(r_{6} \times e_{6}\right)^{T}
\end{array}\right]\left[\begin{array}{c}
V \\
\omega
\end{array}\right] .
$$

Shorthand for

$$
[v]=[J]\left[\begin{array}{ll}
V & \omega
\end{array}\right]^{T}
$$

where $[J]$ is the Jacobian matrix.

2.2.2. Structural Dynamic Equation. Let $m$ be the mass of the upper platform and $I_{a}$ be the moment of inertia of the upper platform. Because the mass and inertia of each leg are relatively small on the upper and lower platforms, it is unnecessary to consider. The electrohydraulic equivalent stiffness in the six supporting legs is determined by $k_{1}, k_{2}, \ldots$, $k_{6}$, damping is expressed by $c_{1}, c_{2}, \ldots, c_{6}$, and the output force is $f_{a 1}, f_{a 2}, \ldots, f_{a 6}$ denotes.

The virtual power of the inertial force acting on the body is calculated, which can be obtained according to the virtual power principle:

$$
\begin{aligned}
\delta p= & \left(f_{a}-k \Delta l-c l\right) \cdot \delta \dot{l}+(-m \ddot{p}) \cdot \delta \dot{p} \\
& +\left(-I_{a} \dot{\omega}-\omega \times I_{a} \omega\right) \cdot \delta \omega=0 .
\end{aligned}
$$

Because the working space of the electrohydraulic Stewart platform is very small, Coriolis and centrifugal forces can be ignored. Thus, (13) can be reduced to

$$
\delta p=\left(f_{a}-k J x-c J \dot{x}\right)^{T} J \delta \dot{x}-\left(m \ddot{p} I_{a} \dot{\omega}\right)^{T} \delta \dot{x}=0 .
$$

In the above formula, $\dot{x}=(\dot{p} \omega)^{T}$. Since the components of $\delta \dot{x}$ are independent of each other, it means that all the coefficients are zero, and the explicit dynamic equation is finally obtained:

$$
M \ddot{x}+C \dot{x}+K x=J^{T} f_{a},
$$

where

$$
\begin{aligned}
M & =\left[\begin{array}{rr}
m I^{3 \times 3} & 0 \\
0 & I_{a}
\end{array}\right], \\
K & =J^{T} k J, \\
C & =J^{T} c J, \\
k & =\operatorname{diag}\left(\left[\begin{array}{llllll}
k_{1} & k_{2} & k_{3} & k_{4} & k_{5} & k_{6}
\end{array}\right]\right), \\
c & =\operatorname{diag}\left(\left[\begin{array}{llllll}
c_{1} & c_{2} & c_{3} & c_{4} & c_{5} & c_{6}
\end{array}\right]\right), \\
f_{a} & =\left(\begin{array}{llllll}
f_{a 1} & f_{a 2} & f_{a 3} & f_{a 4} & f_{a 5} & f_{a 6}
\end{array}\right)^{T} .
\end{aligned}
$$

\section{Dynamic Equivalent Stiffness of Fluid Element}

3.1. Theoretical Model of Dynamic Bulk Modulus of Elasticity. Generally, the transmission medium of the electrohydraulic Stewart platform is gas-oil, which belongs to gas-liquid mixed fluid. The transmission medium is regarded as an elastomer, and its definition is

$$
E=-V \frac{\Delta p}{\Delta V}
$$

where $V$ is the total volume of gas-liquid mixture $\left(\mathrm{m}^{3}\right), \Delta p$ is the change in pressure of gas-liquid mixture $(\mathrm{Pa})$, and $\Delta V$ is the volume change of gas-liquid mixture $\left(\mathrm{mm}^{3}\right)$.

Take the small pressure change, and then the differential expression of the above equation is as follows: 


$$
E=-V \frac{\mathrm{d} p}{\mathrm{~d} V}
$$

\subsection{Dynamic Equivalent Stiffness of the Leg Hydraulic Cyl-} inder Fluid Unit. In the Stewart platform system stiffness matrix, the stiffness of the fluid element in the leg hydraulic cylinder needs to be equivalent calculated. Based on the theory of dynamic volumetric elastic modulus of gas-liquid mixed fluid, the dynamic equivalent stiffness of each leg fluid element of the Stewart platform system is calculated.

Let the average cross-sectional area of the fluid unit be $S_{a}$ and the axial length $l$, then the volume of the fluid unit in the definition of the volume elastic modulus of the gas-liquid mixed fluid as shown in (17) can be expressed as

$$
V=S_{a} l
$$

The stiffness of an elastomer is defined as

$$
k=-\frac{\Delta F}{\Delta l}
$$

where $\Delta F$ is the change in fluid unit load $(\mathrm{N})$ and $\Delta l$ is the variation in the axial length of a fluid element $\left(\mathrm{mm}^{3}\right)$.

By combining (17)-(20), the dynamic equivalent stiffness of fluid element expressed by dynamic volume elastic modulus of gas-liquid mixed fluid can be obtained as

$$
k=\frac{E S_{a}}{l} .
$$

\section{Modal Analysis of Electrohydraulic Stewart Platform}

4.1. Modal Analysis of the Electrohydraulic Stewart Platform at Pose 1. The hydraulic stiffness of six legs is divided into two different cases to study (unit: $\mathrm{N} / \mathrm{m}$ ):

$$
\begin{aligned}
& \text { Case (a): } k_{1}=6 \times 10^{5}, \quad k_{2}=6 \times 10^{5}, \quad k_{3}=6 \times 10^{5}, \\
& k_{4}=6 \times 10^{5}, k_{5}=6 \times 10^{5}, k_{6}=6 \times 10^{5} . \\
& \text { Case (b): } k_{1}=6 \times 10^{5}, \quad k_{2}=5.9 \times 10^{5}, \quad k_{3}=5.8 \times 10^{5}, \\
& k_{4}=5.7 \times 10^{5}, k_{5}=5.6 \times 10^{5}, k_{6}=5.5 \times 10^{5} .
\end{aligned}
$$

By substituting the pose parameters of pose 1, data in Table 1, and hydraulic stiffness of six legs into (15), the modal frequency and mode shapes of the platform transmission system can be obtained, as shown in Tables 2 and 3, which correspond to Cases (a) and (b), respectively.

According to Tables 2 and 3, the Stewart 6-DOF platform has the following characteristics:

(1) According to the characteristics of each mode shape, each mode is named as the moving vibration mode and the rotating vibration mode of the upper platform along the $X$-axis, $Y$-axis, and $Z$-axis; that is, in each mode, the vibration displacement and vibration rotation of the upper platform along the $X$-axis, $Y$ axis, and $Z$-axis are the maximum, respectively.

(2) In the positive position, when the hydraulic stiffness of the six supporting legs is the same, the mobile vibration mode and the rotational vibration mode frequency of the upper platform along the $X$-axis and $Y$-axis are the same.

(3) In the positive position, when the six supporting legs have the same hydraulic stiffness, the system only has two coupling modes: the upper platform moving vibration modes along the $X$-axis and the upper platform rotating vibration modes along the $Y$-axis, and the upper platform moving vibration modes along the $Y$-axis and the upper platform rotating vibration modes along the $X$-axis.

(4) In the positive position, when the hydraulic stiffness of the six supporting legs is different, the system has six order different natural frequencies, and the coupling of the system increases.

4.2. Modal Analysis of the Electrohydraulic Stewart Platform at Pose 2. The hydraulic stiffness of six legs is divided into two different cases to study (unit: $\mathrm{N} / \mathrm{m}$ ):

$$
\begin{aligned}
& \text { Case (c): } k_{1}=6 \times 10^{5}, \quad k_{2}=6 \times 10^{5}, \quad k_{3}=6 \times 10^{5}, \\
& k_{4}=6 \times 10^{5}, k_{5}=6 \times 10^{5}, k_{6}=6 \times 10^{5} . \\
& \text { Case (d): } k_{1}=6 \times 10^{5}, \quad k_{2}=5.9 \times 10^{5}, \quad k_{3}=5.8 \times 10^{5}, \\
& k_{4}=5.7 \times 10^{5}, k_{5}=5.6 \times 10^{5}, k_{6}=5.5 \times 10^{5} .
\end{aligned}
$$

By subbing pose parameters of pose 2 , data in Table 1 , and hydraulic stiffness of six legs into (15), the modal frequency and mode shapes of the platform transmission system can be obtained, as shown in Tables 4 and 5, which correspond to Cases (c) and (d), respectively:

According to Tables 2-5, the Stewart 6-DOF platform has the following characteristics:

(1) When the pose of the platform is changed, the Jacobian matrix of the upper platform and the outer leg changes, leading to the change of the stiffness matrix in the system dynamics equation, so the sixth-order natural frequency and the corresponding mode shapes of the system change, but the influence range is small.

(2) As the six leg stiffness decreases, and the platform along the $X$-axis and $Y$-axis and $Z$-axis movement vibration modal and rotating vibration modal and the six degree of freedom in addition to their main characteristics, namely, the platform along the $X$-axis and $Y$-axis and $Z$-axis movement vibration and rotation vibration relative to 1 , still remaining in the modal degree-of-freedom vibration were little changed.

(3) Among the modal frequencies, lateral and longitudinal rotation of the upper platform requires stronger supporting capacity than lateral and longitudinal movement of the upper platform. Therefore, the natural frequencies corresponding to the rotating vibration modes along the $X$ and $Y$ axes of the upper platform are larger than the moving vibration modes along the $X$ and $Y$ axes of the platform. On the contrary, the bearing capacity of 
TABLE 2: Modal frequencies and corresponding modal shapes of the Stewart platform in Case (a).

\begin{tabular}{|c|c|c|c|c|c|c|}
\hline $\begin{array}{l}\text { Vibration modes of each degree of } \\
\text { freedom of upper platform }\end{array}$ & $\begin{array}{l}\text { Moving along } \\
\text { the } X \text {-axis }\end{array}$ & $\begin{array}{l}\text { Moving along } \\
\text { the } Y \text {-axis }\end{array}$ & $\begin{array}{l}\text { Moving along } \\
\text { the } Z \text {-axis }\end{array}$ & $\begin{array}{c}\text { Rotate along } \\
\text { the } X \text {-axis }\end{array}$ & $\begin{array}{l}\text { Rotate along } \\
\text { the } Y \text {-axis }\end{array}$ & $\begin{array}{l}\text { Rotate along } \\
\text { the } Z \text {-axis }\end{array}$ \\
\hline Frequency $(\mathrm{Hz})$ & 20.4484 & 20.4484 & 58.4773 & 83.4761 & 83.4761 & 41.3497 \\
\hline \multirow{6}{*}{ Modal vibration mode } & 1 & 0 & 0 & 0 & -0.1543 & 0 \\
\hline & 0 & 1 & 0 & -0.1543 & 0 & 0 \\
\hline & 0 & 0 & 1 & 0 & 0 & 0 \\
\hline & 0 & -0.1538 & 0 & -1 & 0 & 0 \\
\hline & 0.1538 & 0 & 0 & 0 & 1 & 0 \\
\hline & 0 & 0 & 0 & 0 & 0 & -1 \\
\hline
\end{tabular}

TABle 3: Modal frequencies and corresponding modal shapes of the Stewart platform in Case (b).

\begin{tabular}{lcccccc}
\hline $\begin{array}{l}\text { Vibration modes of each degree of } \\
\text { freedom of upper platform }\end{array}$ & $\begin{array}{c}\text { Moving along } \\
\text { the } X \text {-axis }\end{array}$ & $\begin{array}{c}\text { Moving along } \\
\text { the } Y \text {-axis }\end{array}$ & $\begin{array}{c}\text { Moving along } \\
\text { the } Z \text {-axis }\end{array}$ & $\begin{array}{c}\text { Rotate along } \\
\text { the } X \text {-axis }\end{array}$ & $\begin{array}{c}\text { Rotate along } \\
\text { the } Y \text {-axis }\end{array}$ & $\begin{array}{c}\text { Rotate along } \\
\text { the } Z \text {-axis }\end{array}$ \\
\hline Frequency $(\mathrm{Hz})$ & 20.2278 & 20.4584 & 58.1965 & 82.6201 & 83.5604 \\
& 1 & 0.2417 & -0.0110 & 0.0381 & -0.1592 \\
& -0.2417 & 1 & -0.0063 & 0.1504 & 0.0406 & -0.0033 \\
& 0.0075 & 0.0072 & 1 & 0.0140 & -0.0131 & 0.0173 \\
Modal vibration mode & 0.0407 & -0.1488 & -0.0090 & 1 & 0.2700 & 0.0217 \\
& 0.1575 & 0.0383 & 0.0139 & -0.2700 & 1 & 0.0122 \\
& 0.0041 & -0.0050 & 0.0087 & 0.0088 & 0.0085 & -1 \\
\hline
\end{tabular}

TABLE 4: Modal frequencies and corresponding modal shapes of the Stewart platform in Case (c).

\begin{tabular}{|c|c|c|c|c|c|c|}
\hline $\begin{array}{l}\text { Vibration modes of each degree of } \\
\text { freedom of upper platform }\end{array}$ & $\begin{array}{l}\text { Moving along } \\
\text { the } X \text {-axis }\end{array}$ & $\begin{array}{l}\text { Moving along } \\
\text { the } Y \text {-axis }\end{array}$ & $\begin{array}{l}\text { Moving along } \\
\text { the } Z \text {-axis }\end{array}$ & $\begin{array}{c}\text { Rotate along } \\
\text { the } X \text {-axis }\end{array}$ & $\begin{array}{c}\text { Rotate along } \\
\text { the } Y \text {-axis }\end{array}$ & $\begin{array}{c}\text { Rotate along } \\
\text { the } Z \text {-axis }\end{array}$ \\
\hline \multirow[t]{3}{*}{ Frequency $(\mathrm{Hz})$} & 20.1335 & 20.7141 & 58.4720 & 81.2448 & 84.9425 & 40.9946 \\
\hline & 1 & 0.5786 & -0.0038 & 0.1159 & -0.1447 & -0.0592 \\
\hline & 0.5792 & -1 & 0.0091 & -0.1617 & -0.1058 & -0.0288 \\
\hline \multirow{4}{*}{ Modal vibration mode } & 0.0021 & -0.0116 & -1 & 0.0003 & -0.0016 & 0.0221 \\
\hline & -0.08 & 0.1642 & -0.0007 & -1 & -0.6944 & 0.0313 \\
\hline & 0.1466 & 0.0918 & -0.0024 & -0.6944 & 1 & -0.0613 \\
\hline & -0.0437 & -0.0031 & -0.011 & 0.0046 & -0.0359 & -1 \\
\hline
\end{tabular}

TABLE 5: Modal frequencies and corresponding modal shapes of the Stewart platform in Case (d).

\begin{tabular}{|c|c|c|c|c|c|c|}
\hline $\begin{array}{l}\text { Vibration modes of each degree of } \\
\text { freedom of upper platform }\end{array}$ & $\begin{array}{l}\text { Moving along } \\
\text { the } X \text {-axis }\end{array}$ & $\begin{array}{l}\text { Moving along } \\
\text { the } Y \text {-axis }\end{array}$ & $\begin{array}{l}\text { Moving along } \\
\text { the } Z \text {-axis }\end{array}$ & $\begin{array}{l}\text { Rotate along } \\
\text { the } X \text {-axis }\end{array}$ & $\begin{array}{c}\text { Rotate along } \\
\text { the } Y \text {-axis }\end{array}$ & $\begin{array}{c}\text { Rotate along } \\
\text { the } Z \text {-axis }\end{array}$ \\
\hline Frequency $(\mathrm{Hz})$ & 19.6809 & 20.294 & 57.2292 & 79.4651 & 83.2042 & 40.1239 \\
\hline \multirow{6}{*}{ Modal vibration mode } & 1 & -0.3536 & 0.0068 & -0.1017 & 0.1476 & -0.0553 \\
\hline & 0.3554 & 1 & 0.0154 & 0.1572 & 0.083 & -0.0372 \\
\hline & 0.0105 & 0.0128 & -1 & 0.0013 & 0.0241 & 0.033 \\
\hline & -0.0388 & -0.1592 & 0.0097 & 1 & 0.5615 & 0.052 \\
\hline & 0.1524 & -0.0591 & -0.0174 & 0.5604 & -1 & -0.0473 \\
\hline & -0.0387 & -0.0114 & -0.0163 & 0.0127 & 0.0331 & -1 \\
\hline
\end{tabular}

the supporting leg required by the vertical rotation of the upper platform is weaker than that required by the vertical movement of the upper platform. Therefore, the natural frequency value corresponding to the rotating vibration mode of the upper platform along the $Z$-axis is smaller than that of the moving vibration mode of the upper platform along the $X$-axis.

(4) Among the modal frequencies, the vertical movement of the upper platform requires a stronger bearing capacity than the lateral and longitudinal movement of the upper platform. Therefore, the natural frequency value corresponding to the moving vibration modes along the $Z$-axis of the upper platform is larger than that of the moving vibration modes along the $X$-axis and $Y$-axis of the upper platform. On the contrary, the vertical rotation of the upper platform requires a weaker bearing capacity than the lateral and longitudinal rotation of the upper platform. Therefore, the natural frequency value corresponding to the $Z$-axis rotational vibration mode of the upper platform is smaller than that of the $X$-axis and $Y$-axis rotational vibration modes of the upper platform. 


\section{Sensitivity Analysis of Natural Frequency of Electrohydraulic Stewart Platform}

5.1. Derivation of Natural Frequency Sensitivity Formula of Transmission System. The eigenvalue equation of (15) is

$$
\left(k-\omega_{i}^{2} m\right) \phi_{i}=0,
$$

where $\phi_{i}$ is the mode shape vector of the Stewart platform.

(22) is left multiplied by $\phi_{i}^{T}$ to get

$$
\phi_{i}^{T}\left(k-\omega_{i}^{2} m\right) \phi_{i}=0 .
$$

If $\omega_{i}^{2}$ is the isolated eigenvalue of the driveline, then it is differentiable with respect to the design variable, so the first derivative of $\omega_{i}$ with respect to the design variable can be obtained by (23). Assuming that the design variable is $t, \omega_{i}, k$, and $\phi_{i}$ are differentiable functions, take the partial derivatives of both sides of (23) with respect to the design variable $t$, and get

$$
\begin{aligned}
\frac{\partial \phi_{i}^{T}}{\partial t}\left(k-\omega_{i}^{2} m\right) \phi_{i} & +\phi_{i}^{T}\left(\frac{\partial k}{\partial t}-\frac{\partial\left(\omega_{i}^{2} m\right)}{\partial t}\right) \phi_{i} \\
& +\phi_{i}^{T}\left(k-\omega_{i}^{2} m\right) \frac{\partial \phi_{i}}{\partial t}=0 .
\end{aligned}
$$

Considering eigenvalue (22), $\left(k-\omega_{i}^{2} m\right)$ is a real symmetric matrix, so $\phi_{i}^{T}$ is the left eigenvector of $\left(k-\omega_{i}^{2} m\right)$, namely,

$$
\phi_{i}^{T}\left(k-\omega_{i}^{2} m\right)=0 .
$$

Substituting (22) and (25) into (24), the simplified formula can be obtained:

$$
\phi_{i}^{T}\left(\frac{\partial k}{\partial t}-2 \omega_{i} \frac{\partial \omega_{i}}{\partial t} m-\omega_{i}^{2} \frac{\partial m}{\partial t}\right) \phi_{i}=0 .
$$

From (26), the sensitivity formula of natural frequency $\omega_{i}$ to design variable $t$ (mass or stiffness parameter of the transmission system) can be obtained:

$$
\frac{\partial \omega_{i}}{\partial t}=\frac{1}{2 a_{e i}}\left(\frac{1}{\omega_{i}} \phi_{i}^{T} \frac{\partial k}{\partial t} \phi_{i}-\omega_{i} \phi_{i}^{T} \frac{\partial m}{\partial t} \phi_{i}\right),
$$

where $a_{e i}=\phi_{i}^{T} m \phi_{i}, \partial k / \partial t$, and $\partial m / \partial t$ are determined by specific design parameters.

In any mode, the derivative of the mass matrix $\mathbf{m}$ with respect to the stiffness parameter is equal to zero, i.e., $(\partial m / \partial t)=0$. Therefore, the sensitivity calculation formula of natural frequency to stiffness parameters is changed into

$$
\frac{\partial \omega_{i}}{\partial t}=\frac{1}{2 a_{e i}}\left(\frac{1}{\omega_{i}} \phi_{i}^{T} \frac{\partial k}{\partial t} \phi_{i}\right) .
$$

For natural frequencies of different modes, the derivative value of stiffness matrix $\mathbf{k}$ with respect to mass parameters is equal to zero; that is, $(\partial k / \partial t)=0$. Therefore, the sensitivity calculation formula of natural frequency with respect to mass parameters is

$$
\frac{\partial \omega_{i}}{\partial t}=-\frac{1}{2 a_{e i}}\left(\omega_{i} \phi_{i}^{T} \frac{\partial m}{\partial t} \phi_{i}\right) .
$$

5.1.1. Sensitivity Formula of Natural Frequency to Mass Parameters. Since changes in the mass of the upper platform affect the system mass matrix, the sensitivity of natural frequency to mass parameters can be calculated according to (29) as follows:

$$
\frac{\partial \omega_{i}}{\partial \rho_{1}}=-\frac{1}{2 a_{e i}}\left(\omega_{i} \phi_{i}^{T} \frac{\partial m}{\partial m_{1}} \phi_{i}\right) .
$$

5.1.2. Sensitivity Formula of Natural Frequency to Stiffness Parameters. Since the variation of stiffness $K$ of the six legs all affects the stiffness matrix $\mathbf{k}$ of the system, the sensitivity calculation formula of natural frequency to stiffness parameters can be obtained according to (28) as follows:

$$
\frac{\partial \omega_{i}}{\partial Q}=\frac{1}{2 a_{e i}}\left(\frac{1}{\omega_{i}} \phi_{i}^{T} \frac{\partial k}{\partial Q} \phi_{i}\right),
$$

where $Q=k_{1}, k_{2}, k_{3}, k_{4}, k_{5}, k_{6}$.

5.2. Sensitivity Analysis of System Natural Frequency with Typical Design Parameters under Platform Post 1. Through (30) and (31), the natural frequency and sensitivity of the platform under the specified pose are calculated. The pose parameters input are

$$
x=\left(P_{x}, P_{y}, P_{z}, \alpha, \beta, \gamma\right)=\left(\begin{array}{llllll}
0 & 0 & 2 \sqrt{3} & 0 & 0 & 0
\end{array}\right) .
$$

5.2.1. Sensitivity Analysis of Natural Frequency to Upper Platform Mass. Keeping other design parameters of Stewart 6-DOF platform unchanged, the sensitivity variation curve of natural frequency and each modal frequency of the transmission system to the mass of the upper platform is shown in the figure.

As can be seen from Figure 4, the sensitivity of natural frequency to the mass $m$ of the upper platform increases with the increase of the mass of the upper platform, and the sensitivity values of the moving modes of $Y$-axis, rotation modes of $Y$-axis, and rotation modes of $Z$-axis of the upper platform vary greatly. The variation curves of the sensitivity values of the $X$-axis moving modes and the $Y$-axis moving modes and the $X$-axis rotating modes and the $Y$-axis rotating modes coincide with each other, and the variation ranges are basically the same. The sensitivity values of all the supporting legs are negative. According to the definition of sensitivity, the natural frequencies of $Z$-axis moving mode and $Z$-axis rotating mode decrease with the increase of internal rotor mass, and the ranges are basically the same. The natural frequency change curves of $X$-axis moving mode and $Y$-axis moving mode and $X$-axis rotating mode and vibration mode are identical, and the ranges are basically the same. 


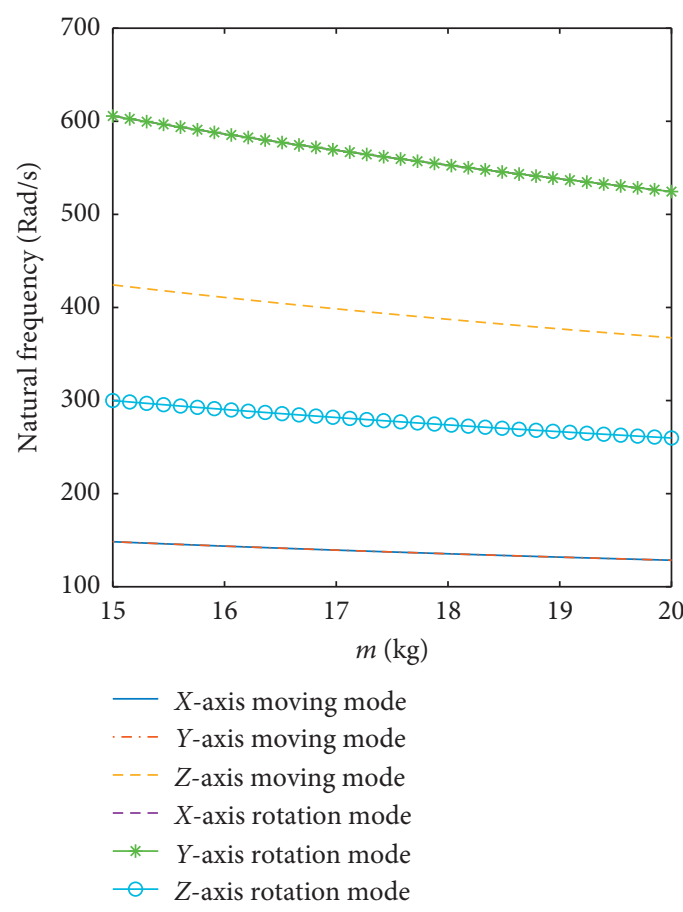

(a)

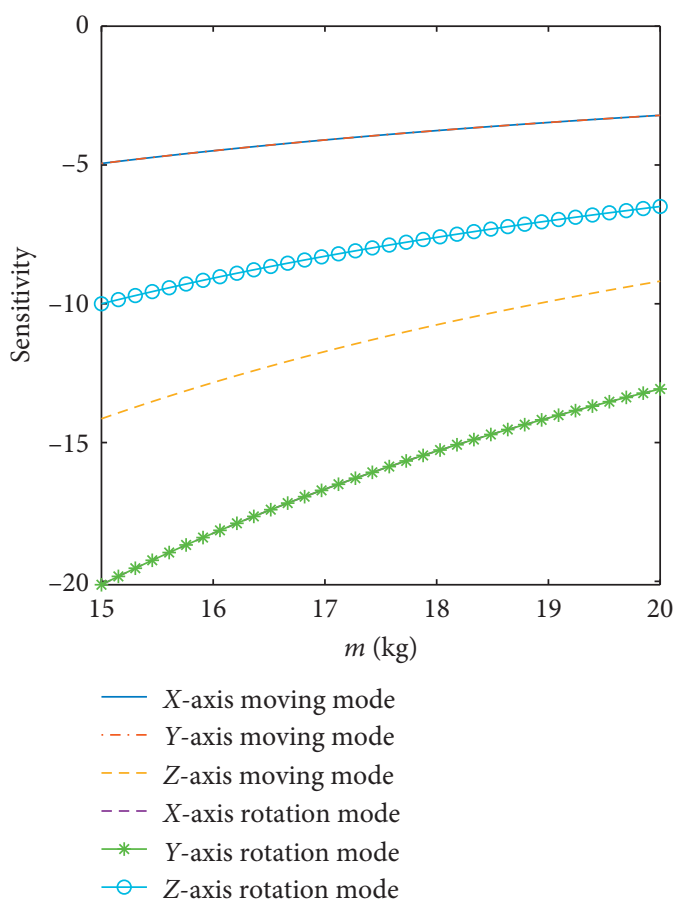

(b)

FIgURE 4: Curves of natural frequency and its sensitivity as a function of upper platform mass. (a) Natural frequency. (b) Sensitivity of natural frequency to upper platform mass.

\subsubsection{Sensitivity Analysis of Natural Frequency to Hydraulic} Stiffness of Outer Leg 1 and Outer Leg 4. According to equation (31), the variation of natural frequency and sensitivity with the stiffness of outer leg 1 is the same as that with the stiffness of outer leg 4. Only the natural frequency and sensitivity of outer leg 1 are analyzed below.

Keeping other design parameters of Stewart 6-DOF platform unchanged, the sensitivity variation curve of natural frequency and each modal frequency of the transmission system to the outer leg 1 is shown in the figure.

Figure 5 shows that the stiffness leg 1 to $6 \times 10^{5} \mathrm{~N} / \mathrm{m}$ and inherent frequency of modal sensitivity with leg $1 X$-axis rotation stiffness increase with the increase of the $k_{a}$, inherent frequency movement modal of $X$-axis and $Z$-axis movement modal, $Z$-axis rotation modal stiffness sensitivity decreases with the increase of the stiffness leg $1 k_{a}$ and $X$-axis motion mode and the $Z$-axis rotation mode, and changes in $Y$-axis motion mode, modal sensitivity almost has no change and the $Y$-axis rotation curves overlap, but when the leg 1 stiffness reaches $6 \times 10^{5} \mathrm{~N} / \mathrm{m}, X$-axis rotation, the sensitivity of modal fell sharply, and the $X$-axis movement modal sensitivity decreased. The sensitivity of the $Y$-axis rotating mode increases sharply, and that of the $Y$-axis moving mode increases sharply. According to the definition of sensitivity, with the increase of stiffness $k_{a}$, the natural frequencies corresponding to each supporting leg mode almost do not change.

5.2.3. Sensitivity Analysis of Natural Frequency to the Hydraulic Stiffness of Outer Leg 2 and Outer Leg 3. According to equation (31), the variation of natural frequency and sensitivity with the stiffness of outer leg 2 is the same as that with the stiffness of outer leg 3 . In the following, only the natural frequency and sensitivity of outer leg 2 are analyzed.

Keeping other design parameters of Stewart 6-DOF platform unchanged, the sensitivity variation curve of natural frequency and each modal frequency of the transmission system to the outer leg 2 is shown in the figure.

Figure 6 shows that the inherent frequency of $Y$-axis rotation modal stiffness sensitivity with leg 2 stiffness increases with the increase of the $\mathrm{kB}$ inherent frequency of leg $Y$ modal and $Z$-axis movement modal, $Z$-axis rotation modal sensitivity decreases with the increase of the stiffness leg $2 \mathrm{kB}$, greatly reduces the sensitivity of the $Y$-axis movement mode, $Y$-axis rotation mode corresponding to sensitivity significantly increased and the value is bigger, the $X$-axis motion mode and $X$-axis rotation mode in leg 2 stiffness sensitivity at $6 \times 105 \mathrm{~N} / \mathrm{m}$ before almost have no change and basic coincidence, and sensitivity curve in leg 2 stiffness reaches $6 \times 105 \mathrm{~N} / \mathrm{m}$. The sensitivity of $X$-axis moving mode increases sharply, and the sensitivity of $X$-axis rotating mode increases somewhat. According to the definition of sensitivity, with the increase of stiffness $\mathrm{kB}$, the natural frequencies corresponding to each supporting leg mode almost did not change.

5.2.4. Sensitivity Analysis of Natural Frequency to the Hydraulic Stiffness of Outer Leg 5 and Outer Leg 6. According to (31), the variation of natural frequency and sensitivity with the stiffness of outer leg 5 is the same as that with the stiffness of outer leg 6 . In the following, only the natural frequency and sensitivity of outer leg 5 are analyzed. 

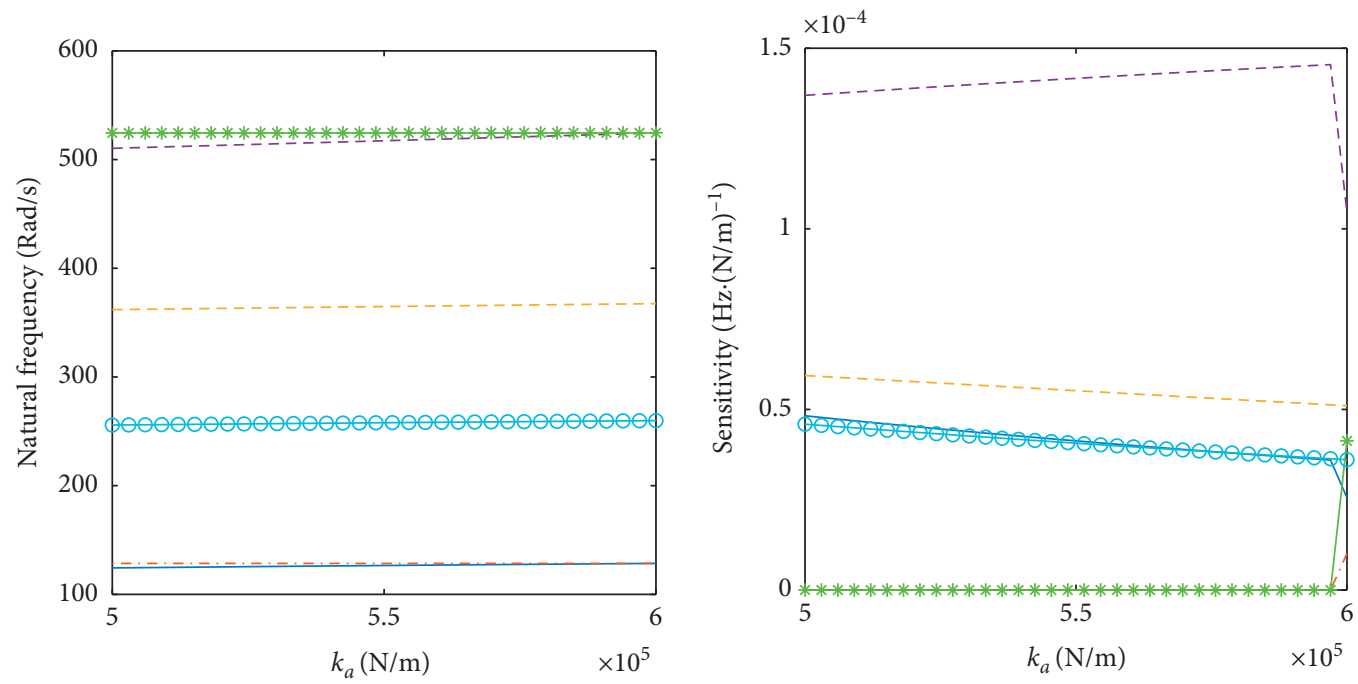

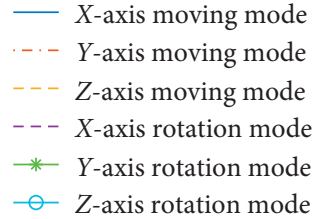

(a)
- $X$-axis moving mode

-.- $Y$-axis moving mode

- - $Z$-axis moving mode

- - - $X$-axis rotation mode

* $Y$-axis rotation mode

$-Z$-axis rotation mode

(b)

FIGURE 5: Curve of natural frequency and its sensitivity changing with the stiffness of outer leg 1. (a) Natural frequency. (b) Sensitivity of natural frequency to the stiffness of outer leg 1.

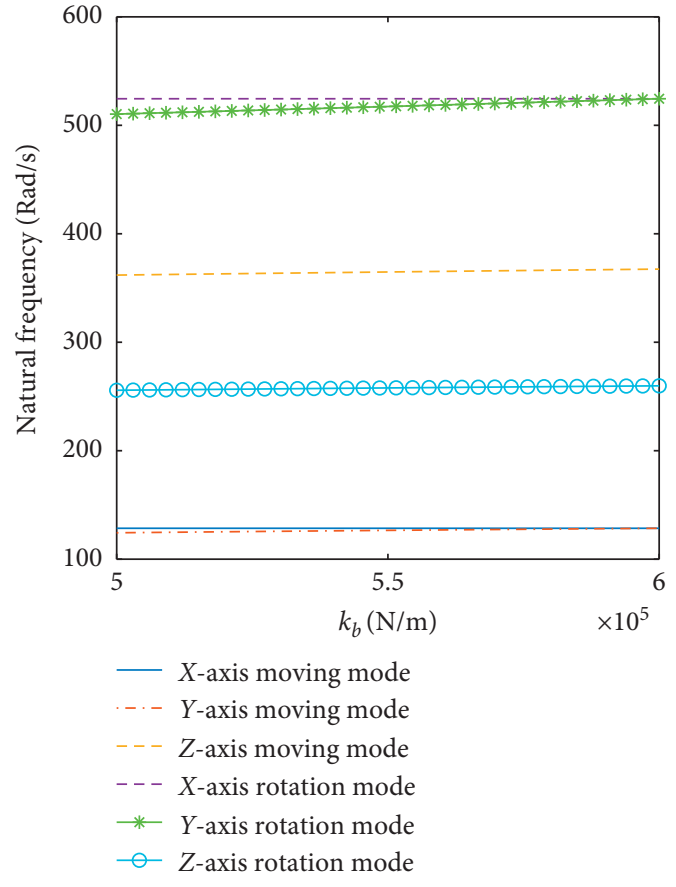

(a)

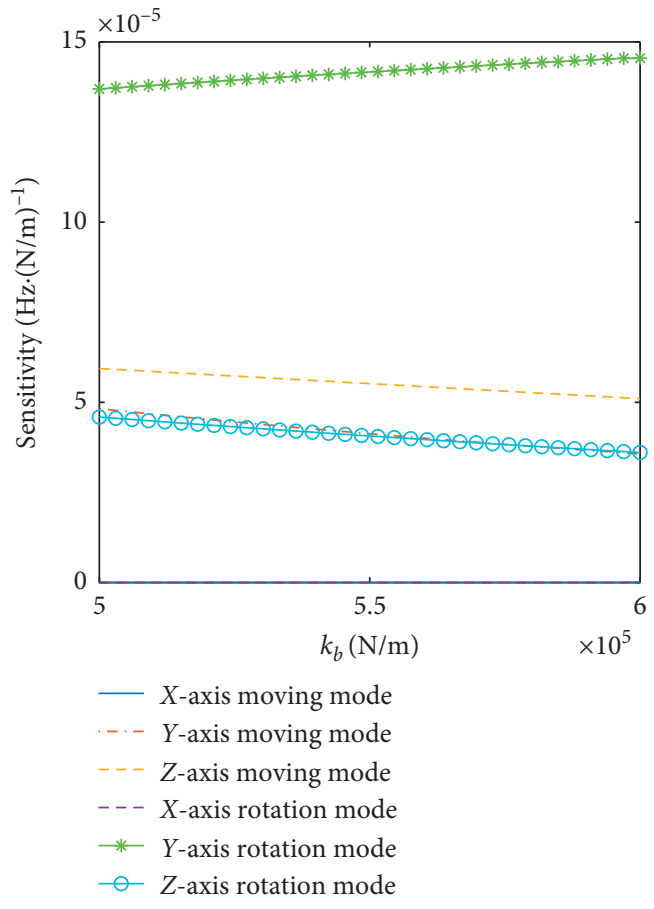

(b)

FIGURE 6: Curve of natural frequency and its sensitivity changing with the stiffness of outer leg 2. (a) Natural frequency. (b) Sensitivity of natural frequency to the stiffness of outer leg 2 . 
Keeping other design parameters of Stewart 6-DOF platform unchanged, the sensitivity variation curve of natural frequency and each modal frequency of the transmission system to the outer leg 5 is shown in the figure.

Figure 7 shows that the stiffness leg 5 to $6 \times 105 \mathrm{~N} / \mathrm{m}$ before the natural frequency of the $X$-axis rotation modal stiffness sensitivity with leg may increase with the increase of the stiffness $k_{e}$, inherent frequency movement modal of $X$ axis and $Z$-axis movement modal, $Z$-axis rotation modal sensitivity decreases with the increase of the leg 5 stiffness $k_{e}$, the $X$-axis motion mode and the $Z$-axis rotation mode, and changes in $Y$-axis motion mode, modal sensitivity almost has no change and the $Y$-axis rotation curves overlap, but when the leg 5 stiffness reaches $6 \times 105 \mathrm{~N} / \mathrm{m}, X$-axis rotation, the sensitivity of modal fell sharply, and the $X$-axis movement modal sensitivity decreased. The sensitivity of the $Y$-axis rotating mode increases sharply, and that of the $Y$-axis moving mode increases sharply. According to the definition of sensitivity, with the increase of stiffness $k_{e}$, the natural frequencies corresponding to each supporting leg mode almost do not change.

5.3. Sensitivity Analysis of System Natural Frequency with Typical Design Parameters under Platform Pose 2. Through (30) and (31), the natural frequency and sensitivity of the platform under the specified pose are calculated. The pose parameters input are as follows:

$$
x=\left(P_{x}, P_{y}, P_{z}, \alpha, \beta, \gamma\right)=\left(\begin{array}{llllll}
1 & 2 & 2 \sqrt{3} & 20 & 15 & 0
\end{array}\right) .
$$

\subsubsection{Sensitivity Analysis of Natural Frequency to Upper} Platform Mass. Keeping other design parameters of Stewart 6-DOF platform unchanged, the sensitivity variation curve of natural frequency and each modal frequency of the transmission system to the mass of the upper platform is shown in the figure.

As can be seen from Figure 8, the sensitivity of natural frequency to the mass $m$ of the upper platform increases with the increase of the mass of the upper platform, and the sensitivity values of $Z$-axis moving mode, $X$-axis rotating mode, and $Y$-axis rotating mode vibration mode vary greatly. The sensitivity values of $X$-axis moving mode, $Y$-axis moving mode, and $Z$-axis rotating mode were basically the same, and all the sensitivity values of the supporting legs were negative. From the definition of sensitivity, it can be seen that the natural frequencies of $Z$-axis moving modes, $X$ axis rotating modes, and $Y$-axis rotating modes decrease with the increase of the internal rotor mass, and the natural frequencies of $X$-axis moving modes, $Y$-axis moving modes and $Z$-axis rotating modes decrease with the increase of the internal rotor mass, and the range is small.

5.3.2. Sensitivity Analysis of Natural Frequency to Hydraulic Stiffness of Outer Leg 1. Keeping other design parameters of Stewart 6-DOF platform unchanged, the sensitivity variation curve of natural frequency and each modal frequency of the transmission system to the outer leg 1 is shown in the figure.
As can be seen from Figure 9, the sensitivity of natural frequency to the $X$-axis rotational mode stiffness increases with the increase of the stiffness $k_{a}$ of the outer leg 1 , while the sensitivity of natural frequency to the $Y$-axis moving mode, $Z$-axis moving mode, and $Z$-axis rotating mode decreases with the increase of the stiffness $k_{a}$ of the outer leg 1 , while the sensitivity of other outer legs almost has no change. According to the definition of sensitivity, with the increase of stiffness $k_{a}$, the natural frequencies corresponding to each supporting leg mode almost do not change.

5.3.3. Sensitivity Analysis of Natural Frequency to Hydraulic Stiffness of Outer Leg 2. Keeping other design parameters of Stewart 6-DOF platform unchanged, the sensitivity variation curve of natural frequency and each modal frequency of the transmission system to the outer leg 2 is shown in the figure.

Figure 10 shows that the inherent frequency of $Y$-axis rotation modal sensitivity increases with the increase of leg 2 $k_{b}$ stiffness, natural frequency movement modal of $X$-axis and $Y$-axis modal, $Z$-axis movement modal, and $Z$-axis rotation modal sensitivity decreases with the increase of the stiffness leg $2 k_{b}, Z$-axis movement mode, greatly reduces the sensitivity of the $Z$-axis rotation mode, $Y$-axis rotation mode corresponding to sensitivity significantly increased, and the value is bigger. According to the definition of sensitivity, with the increase of stiffness $k_{b}$, the natural frequencies corresponding to each supporting leg mode almost did not change.

5.3.4. Sensitivity Analysis of Natural Frequency to Hydraulic Stiffness of Outer Leg 3. Keeping other design parameters of Stewart 6-DOF platform unchanged, the sensitivity variation curve of natural frequency and each modal frequency of the transmission system to the outer leg 3 is shown in the figure.

Figure 11 shows that natural frequency on the $Y$-axis rotation modal sensitivity with leg 3 increases with the increase of the rigidity of $k_{c}$, inherent frequency movement modal of $Y$-axis and $Z$-axis movement modal, $Z$-axis rotation modal sensitivity decreases with the increase of the rigidity leg $3 k_{c}$, which greatly reduces the sensitivity of $Z$ axis movement mode, $Y$-axis rotation mode corresponding to sensitivity significantly increased, and the value is bigger. According to the definition of sensitivity, with the increase of stiffness $k_{c}$, the natural frequencies corresponding to each supporting leg mode almost do not change.

5.3.5. Sensitivity Analysis of Natural Frequency to Hydraulic Stiffness of Outer Leg 4. Keeping other design parameters of Stewart 6-DOF platform unchanged, the sensitivity variation curve of natural frequency and each modal frequency of the transmission system to the outer leg 4 is shown in the figure.

Figure 12 shows that natural frequency on the $Y$-axis rotation modal sensitivity with the increase of the leg 4 stiffness $k_{d}$ increased significantly, the inherent frequency movement modal of $X$-axis and $Y$-axis modal, $Z$-axis movement mode, the $X$-axis rotation mode, the $Z$-axis rotation modal sensitivity with the increase of the stiffness $k_{d}$ 


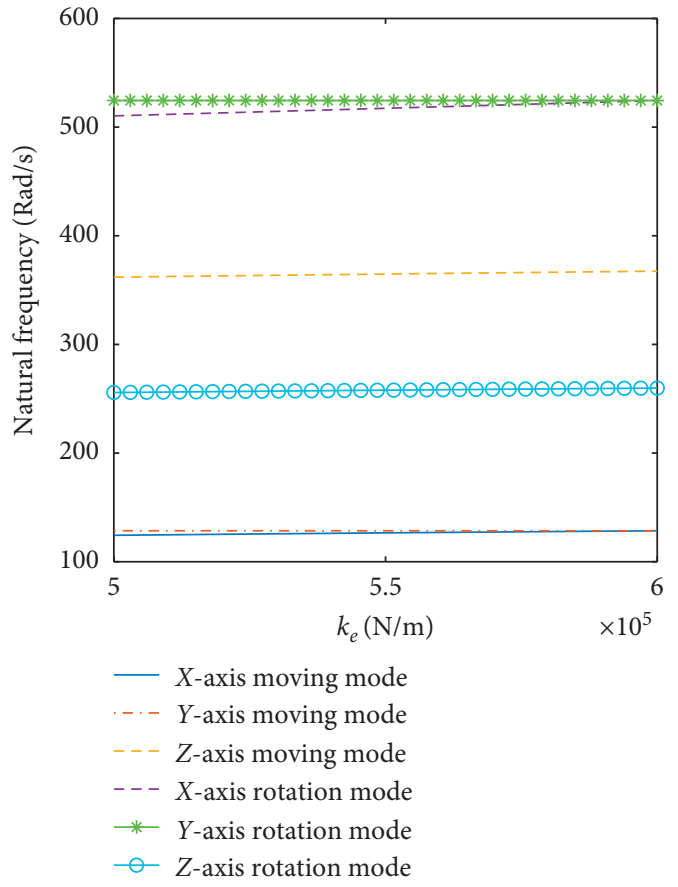

(a)

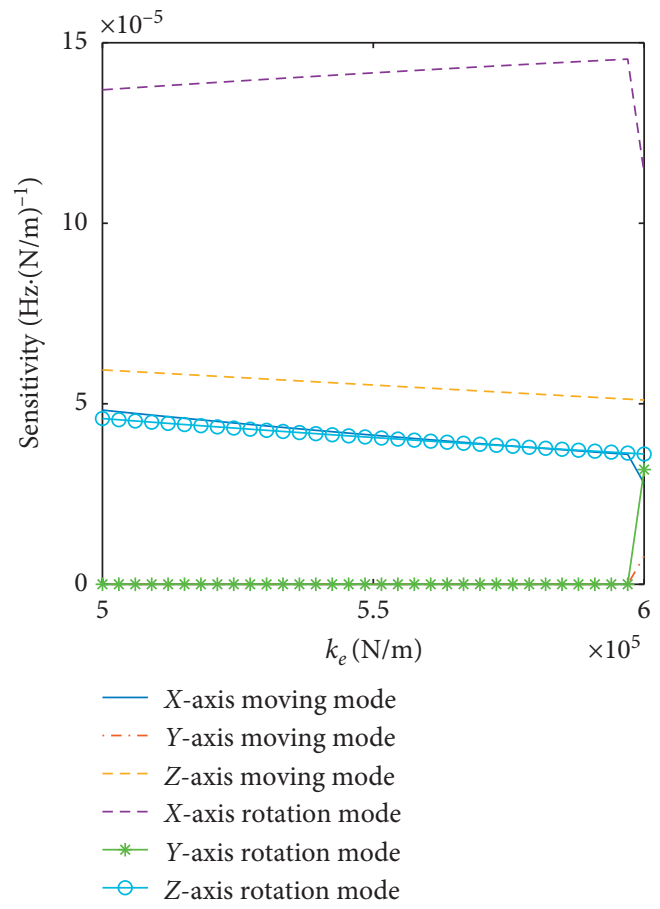

(b)

FIGURE 7: Curve of natural frequency and its sensitivity changing with the stiffness of outer leg 5. (a) Natural frequency. (b) Sensitivity of natural frequency to the stiffness of outer leg 5.

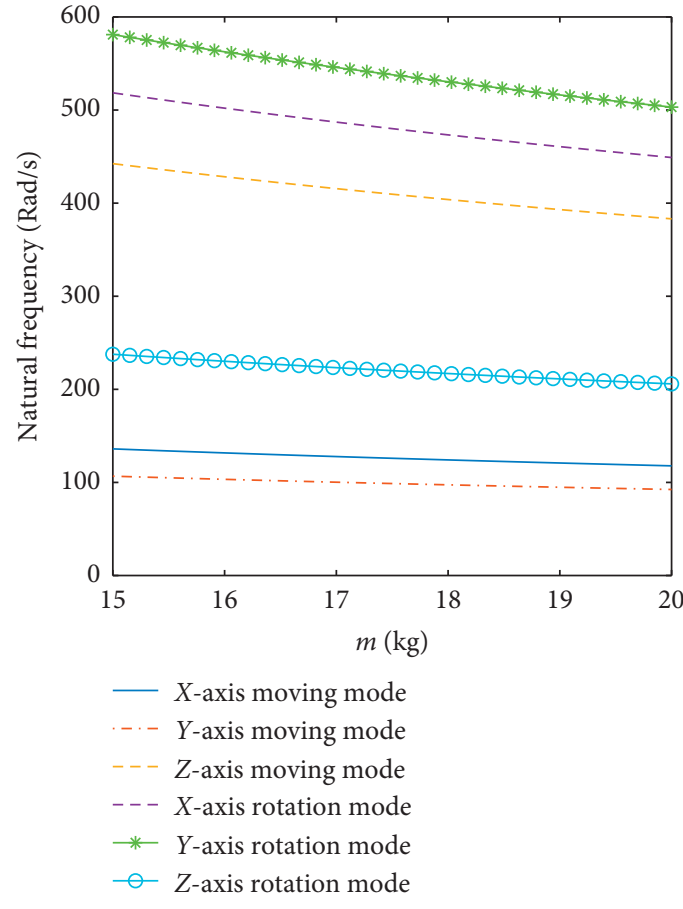

(a)

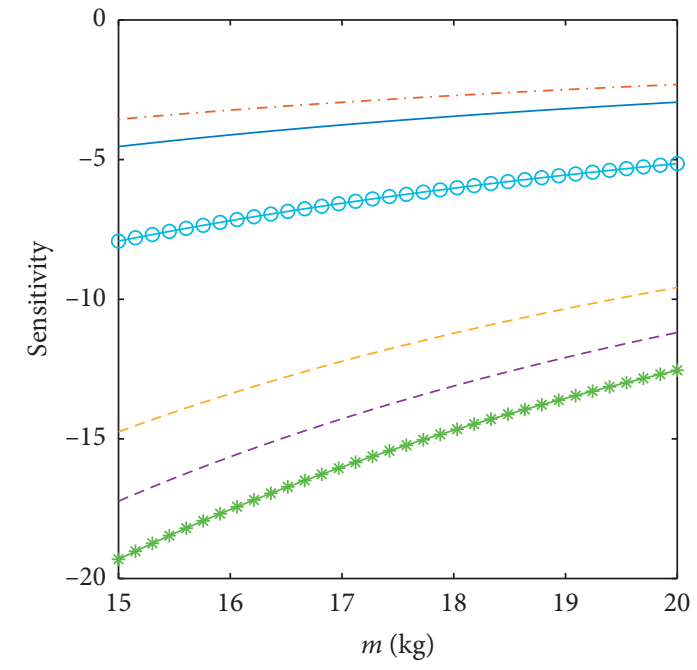

$X$-axis moving mode

... $Y$-axis moving mode

$Z$-axis moving mode

- - $X$-axis rotation mode

* $Y$-axis rotation mode

$\_Z$-axis rotation mode

(b)

Figure 8: Curves of natural frequency and its sensitivity as a function of upper platform mass. (a) Natural frequency. (b) Sensitivity of natural frequency to upper platform mass. 


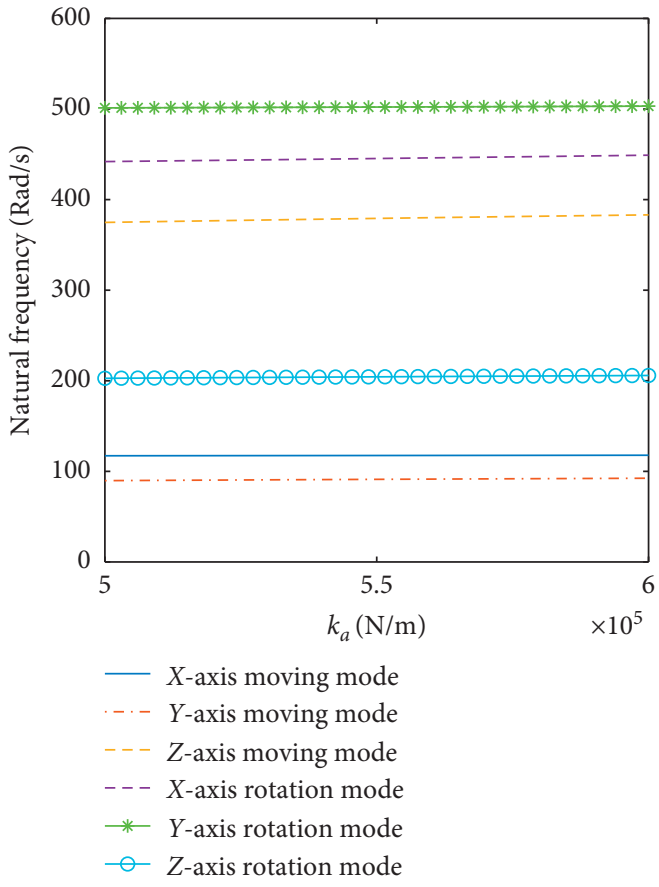

(a)

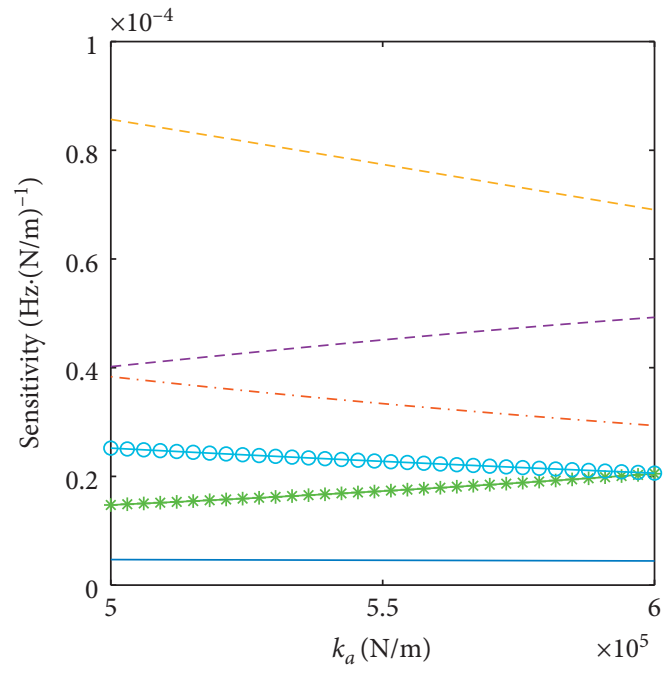

- $X$-axis moving mode

-. $Y$-axis moving mode

--- Z-axis moving mode

- - - $X$-axis rotation mode

* $Y$-axis rotation mode

$-Z$-axis rotation mode

(b)

Figure 9: Curve of variation of natural frequency and its sensitivity with stiffness of outer leg 1. (a) Natural frequency. (b) Sensitivity of natural frequency to the stiffness of outer leg 1.

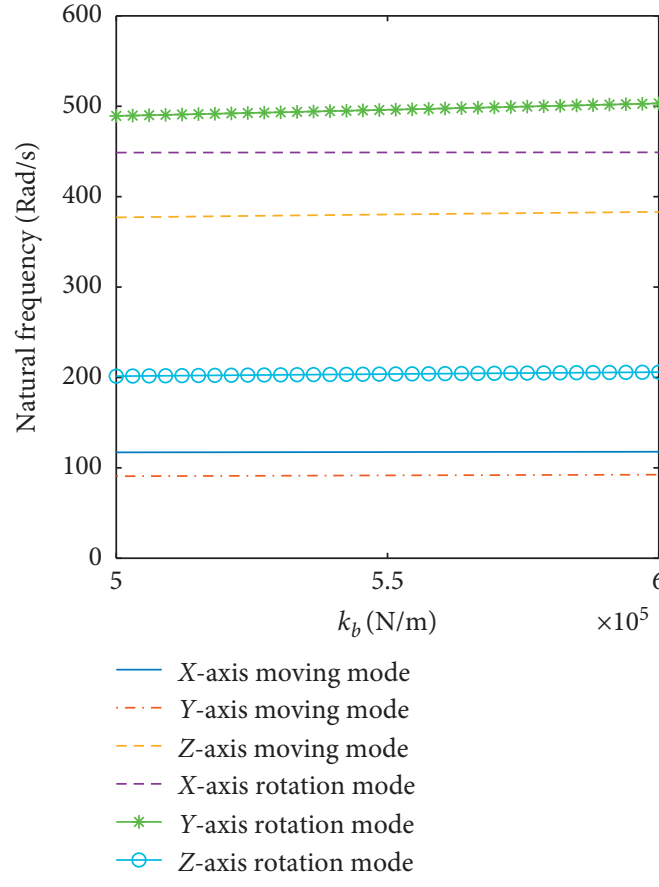

(a)

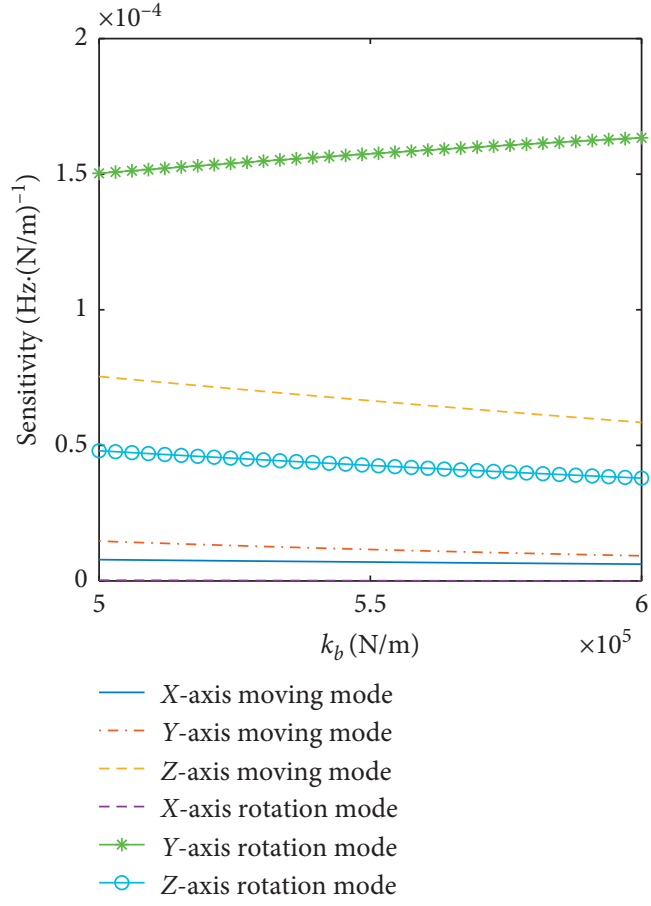

(b)

Figure 10: Curve of variation of natural frequency and its sensitivity with stiffness of outer leg 2. (a) Natural frequency. (b) Sensitivity of natural frequency to the stiffness of outer leg 2. 

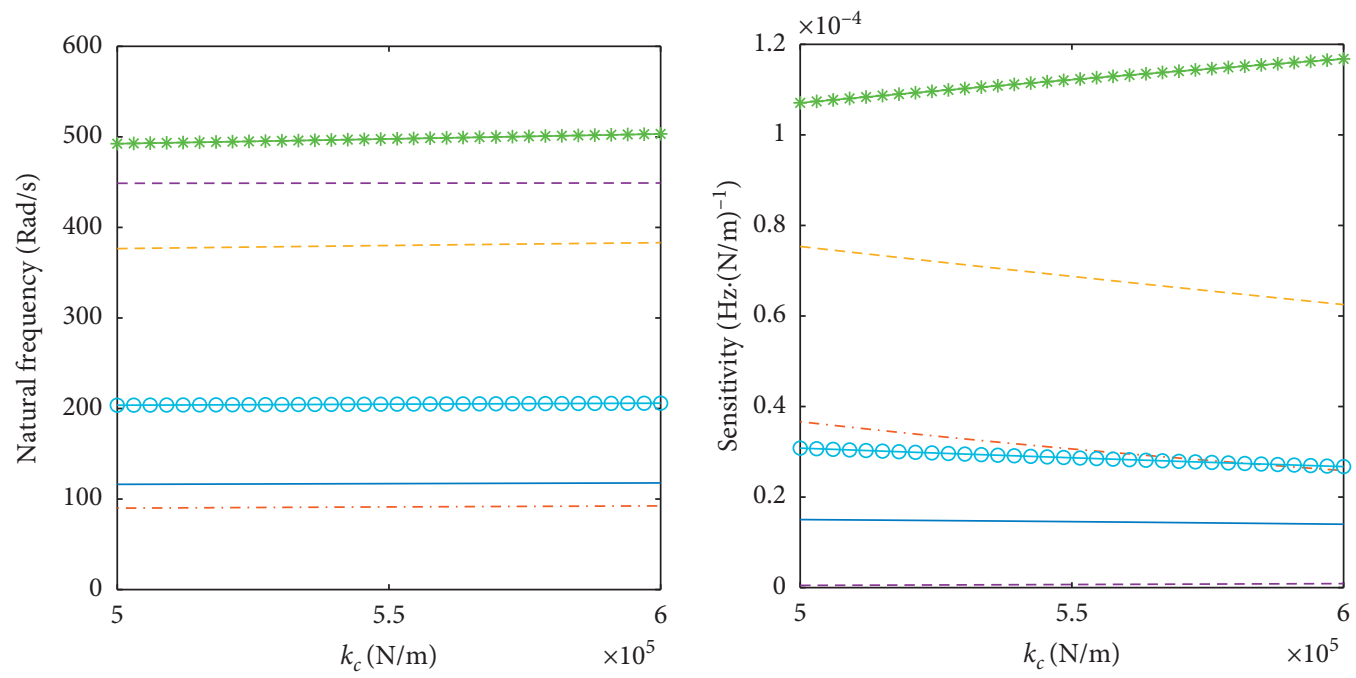

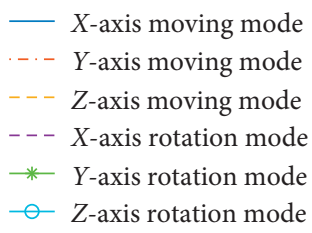

(a)

$$
\begin{aligned}
& -X \text {-axis moving mode } \\
& --Y \text {-axis moving mode } \\
& ---Z \text {-axis moving mode } \\
& ---X \text {-axis rotation mode } \\
& -* \quad Y \text {-axis rotation mode } \\
& -\quad Z \text {-axis rotation mode }
\end{aligned}
$$

(b)

FIgURE 11: Curve of variation of natural frequency and its sensitivity with stiffness of outer leg 3. (a) Natural frequency. (b) Sensitivity of natural frequency to the stiffness of outer leg 3.

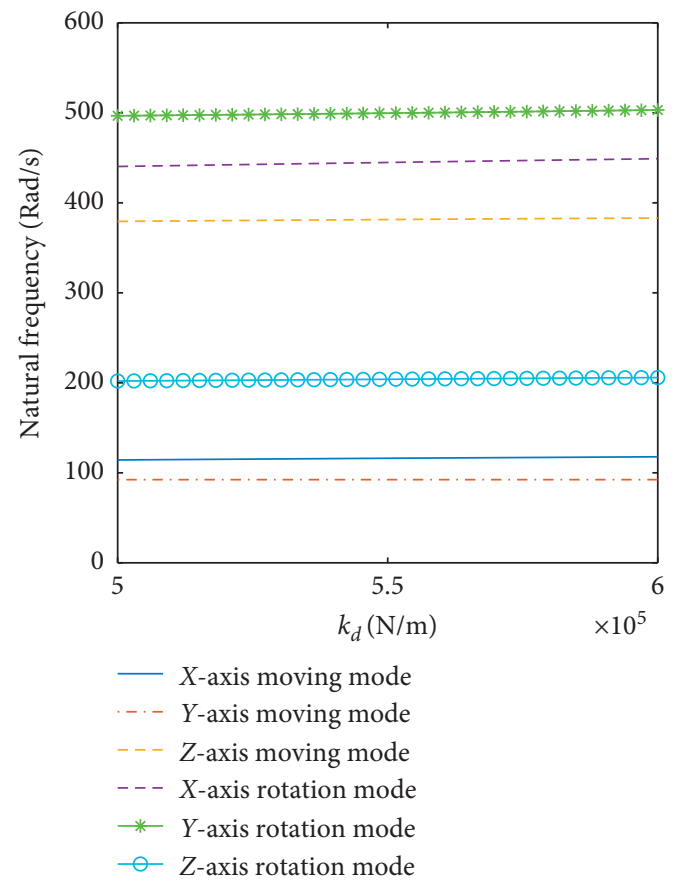

(a)

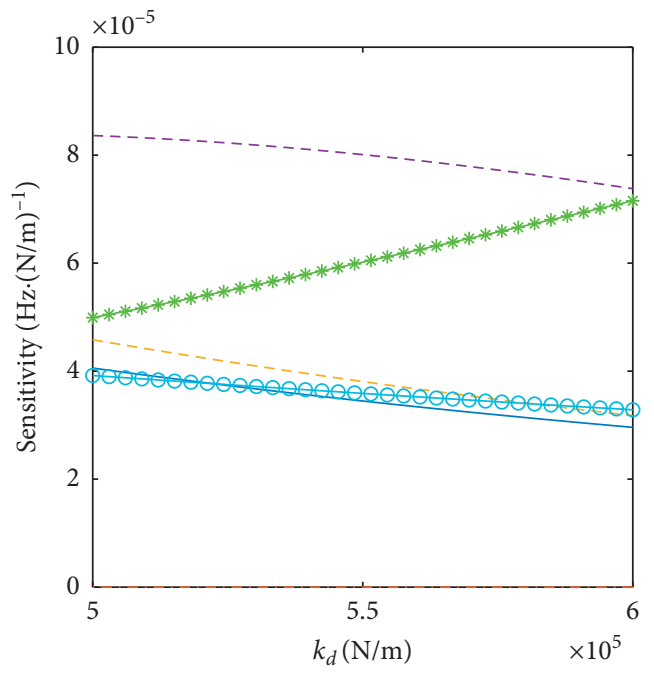

$-X$-axis moving mode
$---Y$-axis moving mode
$---Z$-axis moving mode
$---X$-axis rotation mode
$-\quad Y$-axis rotation mode
$-Z$-axis rotation mode

(b)

FIGURE 12: Curve of variation of natural frequency and its sensitivity with stiffness of outer leg 4. (a) Natural frequency. (b) Sensitivity of natural frequency to the stiffness of outer leg 4 . 
leg 4 significantly decreases, and by the sensitivity definition, with the increase of the stiffness $k_{d}$, each leg modal natural frequency corresponding almost has no change.

5.3.6. Sensitivity Analysis of Natural Frequency to Hydraulic Stiffness of Outer Leg 5. Keeping other design parameters of Stewart 6-DOF platform unchanged, the sensitivity variation curve of natural frequency and each modal frequency of the transmission system to the outer leg 5 is shown in the figure.

Figure 13 shows that natural frequency of the $X$-axis rotation mode and $Y$-axis rotation modal sensitivity with leg 5 increase with the increase of the stiffness $k_{e}$, inherent frequency movement modal of $X$-axis and $Z$-axis rotation modal sensitivity with the increase of the stiffness $k_{e}$ leg 5 significantly decreases, and the other leg sensitivity changes much, and by the sensitivity definition, with the increase of the stiffness $k_{e}$, each leg modal natural frequency corresponding almost has no change.

5.3.7. Sensitivity Analysis of Natural Frequency to $H y$ draulic Stiffness of Outer Leg 6. Keeping other design parameters of Stewart 6-DOF platform unchanged, the sensitivity variation curve of natural frequency and each modal frequency of the transmission system to the outer leg 6 is shown in the figure.

Figure 14 shows that the inherent frequency of $Y$-axis rotation modal sensitivity increases with the increase of leg 6 stiffness $k_{f}$, inherent frequency movement modal of $Y$-axis and $Z$-axis movement modal sensitivity decreases with the increase of the stiffness leg $6 k_{f}$, other leg almost has no change, sensitivity, and by the sensitivity definition, with the increase of the stiffness $k_{f}$, each leg modal natural frequency corresponding almost has no change.

\section{Kinetic Experimental Study}

In this section, the Stewart 6-DOF platform dynamics test bed is built, and the hammering method is used to carry out modal tests on the system. The validity and accuracy of the theoretical analysis results are verified by the experimental value of the first-order natural frequency of the system.

6.1. Construction of the Test Platform. In order to obtain the natural frequency of the Stewart 6-DOF platform, the hammering method was adopted to carry out modal tests on the system under working conditions. The test platform is shown in Figure 15.

The acceleration sensor is installed on the Stewart 6-DOF platform prototype to measure the acceleration signal of the system, and the piezoelectric acceleration sensor is installed to measure the acceleration signal of the upper platform, and the signal analyzer, computer, and sensor are properly connected. The connection diagram of each device in the modal test is shown in Figure 16.

The relevant parameters of the piezoelectric acceleration sensor are shown in Table 6.
6.2. Test and Test Procedures. Specific operations during the modal test are as follows:

(1) Check the Stewart 6-DOF platform prototype, turn on the controller connected to the platform, turn on the pump station, and make the platform move to a certain position and pose.

(2) Install a piezoelectric acceleration sensor, and attach one piezoelectric sensor to one of the legs near the end of the upper platform. The direction of the output end of the sensor is the same as the direction of the legs, and remember the positions of the three directions $X Y Z$ marked on the sensor. Then, another piezoelectric sensor is attached to the circumscribed surface of the upper platform. The direction of the output end of the sensor is the same as the axial direction of the upper platform. Remember the positions of the three directions $X Y Z$ marked on the sensor.

(3) Connect the circuit of the analysis system and knock the platform on the Stewart platform with a force hammer to check whether the analysis system can receive signals normally.

(4) After the above steps are confirmed, increase the system pressure to $5 \mathrm{MPa}$. After the system is stable, keep the system working for $60 \mathrm{~s}$, and record the data of all sensors.

(5) Keep the above conditions unchanged, use the force hammer to repeatedly hit all parts of the platform on the Stewart platform, and record the data of each sensor.

(6) Restore the platform, shut down the pump station and clean the site. Follow the above steps to complete the Stewart 6-DOF platform modal test and collect test data.

\subsection{Analysis of Test Results}

6.3.1. Modal Test Results and Analysis at Pose 1. Fast Fourier transform algorithm is used to transform the acceleration signal of jet system under the action of pulse excitation, and the amplitude-frequency characteristics of the system are obtained.

Based on the modal analysis results of the Stewart 6-DOF platform, Case (a), it is compared with the theoretical calculated values of the Stewart platform in the first-order dynamic natural frequency stabilization stage. The comparison results are shown in Table 7 .

It can be seen from Table 7 that the theoretical calculated value of the first-order natural frequency of the system is very close to the experimental value with a small error, indicating the effectiveness and accuracy of the theoretical analysis of electrohydraulic Stewart platform dynamics.

6.3.2. Modal Test Results and Analysis at Pose 2. Fast Fourier transform algorithm is used to transform the acceleration signal of the jet system under the action of pulse excitation, 

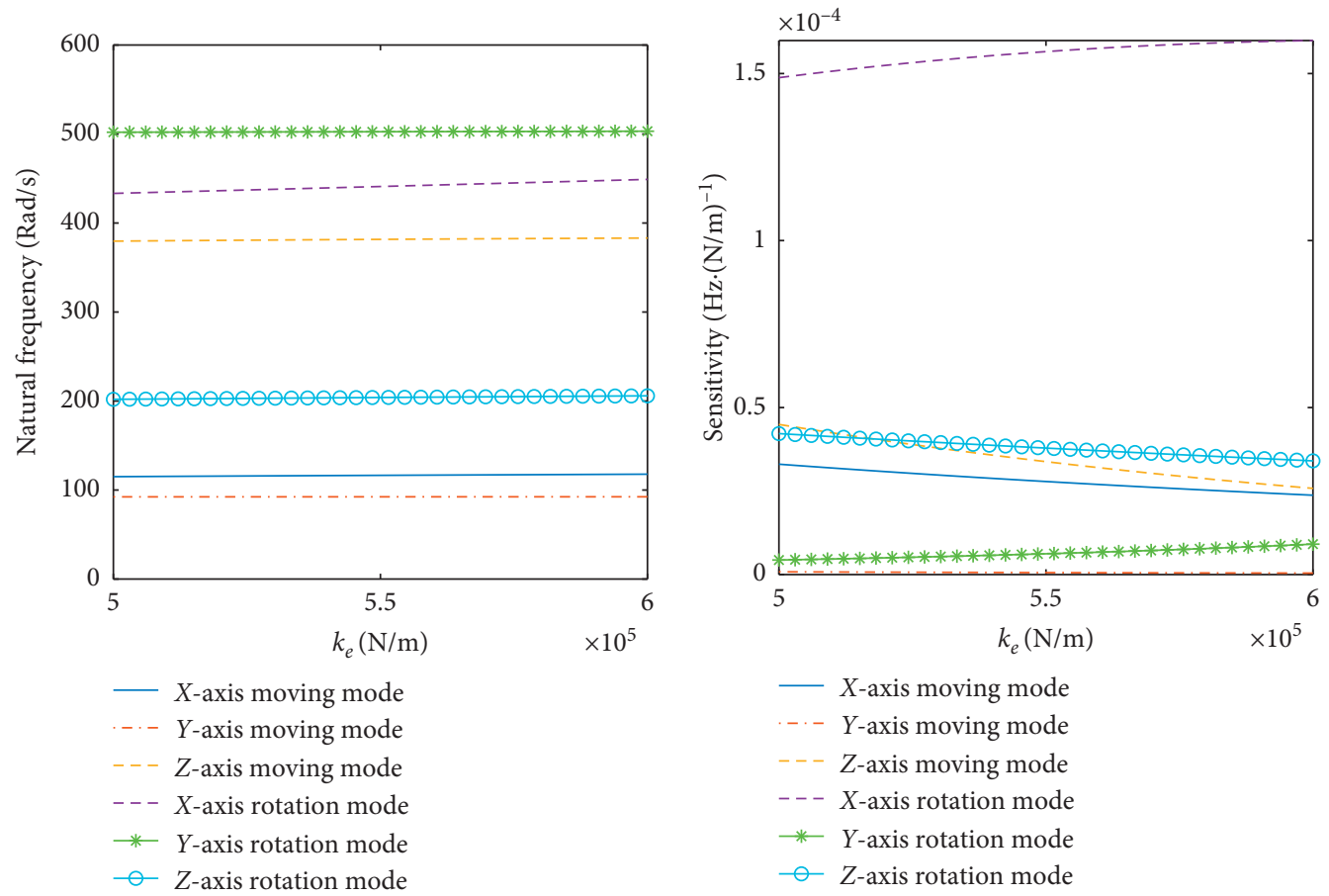

(b)

FIGURE 13: Curve of variation of natural frequency and its sensitivity with stiffness of outer leg 5. (a) Natural frequency. (b) Sensitivity of natural frequency to the stiffness of outer leg 5 .

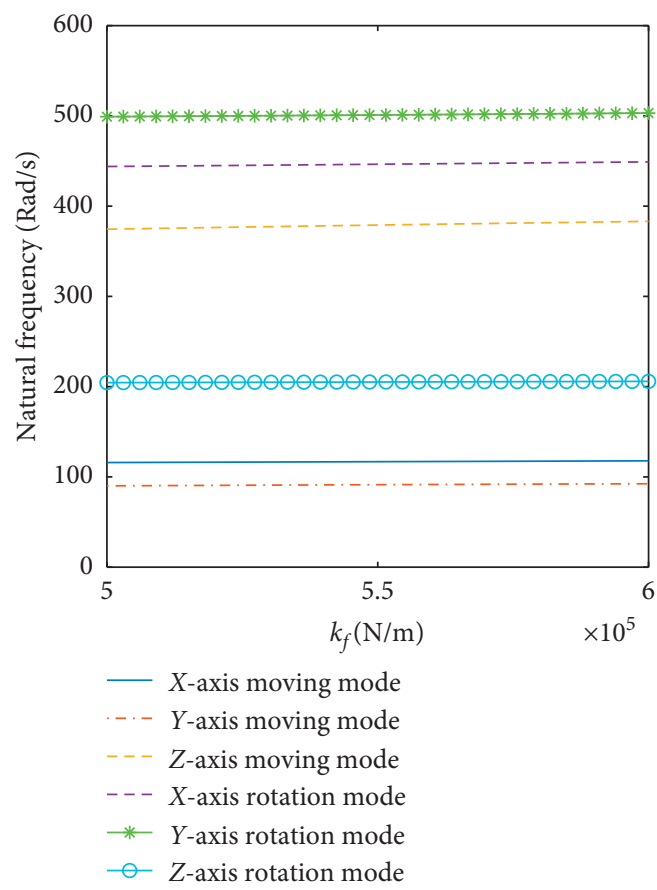

(a)

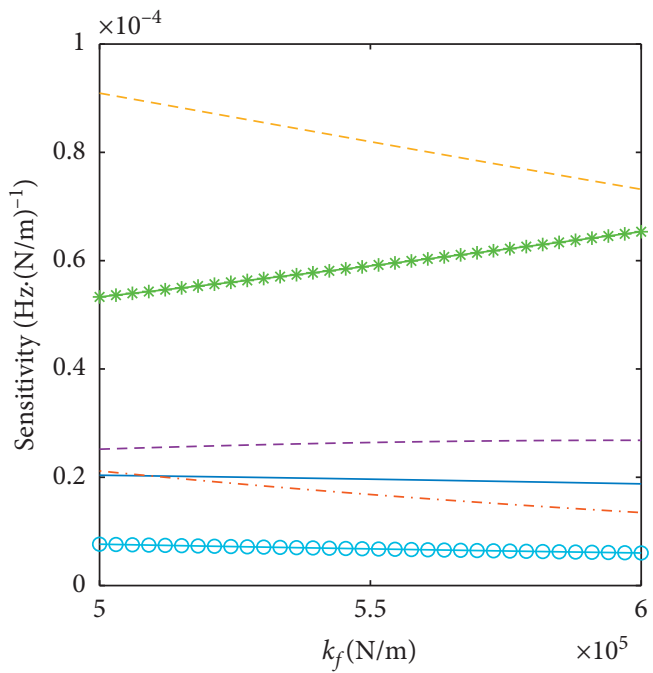

$X$-axis moving mode

-.. $Y$-axis moving mode

- - $Z$-axis moving mode

- - $X$-axis rotation mode

* $Y$-axis rotation mode

$\frown Z$-axis rotation mode

(b)

Figure 14: Curve of variation of natural frequency and its sensitivity with stiffness of outer leg 6. (a) Natural frequency. (b) Sensitivity of natural frequency to the stiffness of outer leg 6. 


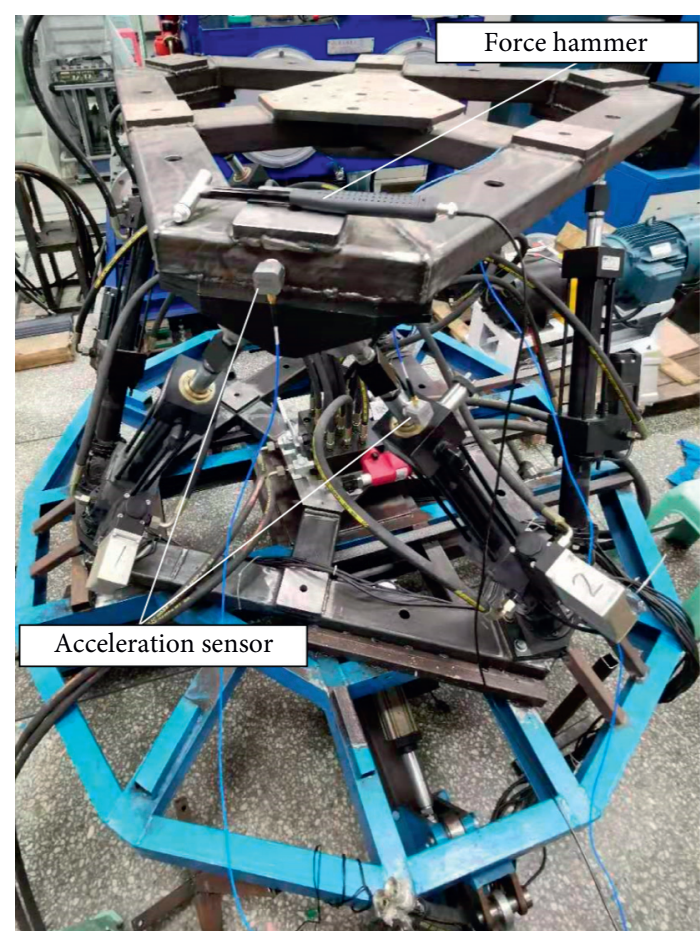

FiguRE 15: Test platform for the modal test.

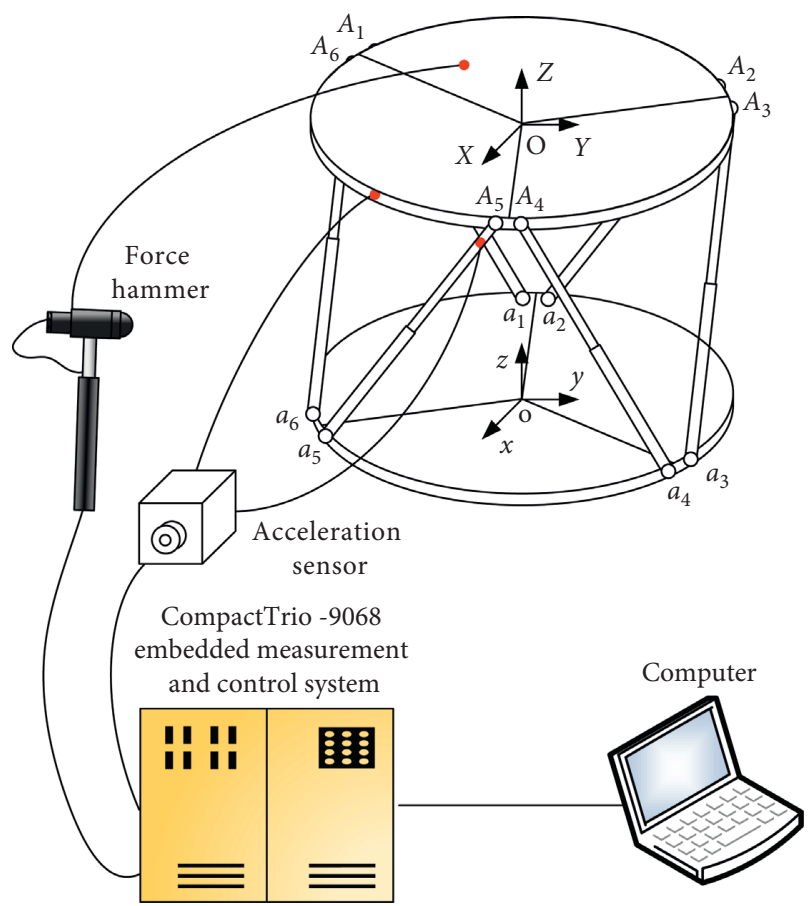

Figure 16: Connecting block diagram of the modal test.

and the amplitude-frequency characteristics of the system are obtained.

Based on the modal analysis results of the Stewart 6-DOF platform, Case (c), it is compared with the theoretical calculated values of the Stewart platform in the first-order dynamic natural frequency stabilization stage. The comparison results are shown in Table 8.
TABLE 6: The relevant parameters of the piezoelectric acceleration sensor.

\begin{tabular}{lc}
\hline Parameter name & Value \\
\hline Sensitivity $\left(\mathrm{mV} \cdot \mathrm{g}^{-1}\right)$ & 100 \\
Frequency response $(\mathrm{Hz})$ & $0.5 \sim 5000$ \\
Scale range $(\mathrm{g})$ & 50 \\
Impact resistant $(\mathrm{g})$ & 200 \\
Excitation voltage $(\mathrm{VDC})$ & $15 \sim 24$ \\
Exciting current $(\mathrm{mA})$ & $2 \sim 10$ \\
\hline
\end{tabular}

TABLE 7: In the condition of pose 1, Case (a), the theoretical value of the first-order natural frequency of the electrohydraulic Stewart platform is compared with the experimental value.

\begin{tabular}{lccc}
\hline $\begin{array}{c}\text { The theoretical value } \\
(\mathrm{Hz})\end{array}$ & $\begin{array}{c}\text { The actual value } \\
(\mathrm{Hz})\end{array}$ & $\begin{array}{c}\text { Error } \\
(\%)\end{array}$ \\
\hline Value & 20.45 & 20.40 & 0.24 \\
\hline
\end{tabular}

TABLE 8: In the condition of pose 2, Case (c), the theoretical value of the first-order natural frequency of the electrohydraulic Stewart platform is compared with the experimental value.

\begin{tabular}{lccc}
\hline & $\begin{array}{c}\text { The theoretical value } \\
(\mathrm{Hz})\end{array}$ & $\begin{array}{c}\text { The actual value } \\
(\mathrm{Hz})\end{array}$ & $\begin{array}{c}\text { Error } \\
(\%)\end{array}$ \\
\hline Value & 20.14 & 20.23 & 4.5 \\
\hline
\end{tabular}

It can be seen from Table 8 that the theoretical calculated value of the first-order natural frequency of the system is close to the experimental value with a small error, indicating the effectiveness and accuracy of the theoretical analysis of electrohydraulic Stewart platform dynamics.

\section{Conclusion}

Based on the above analysis results of natural frequency and sensitivity of the electrohydraulic Stewart 6-DOF platform, the following law can be obtained:

(1) In the same posture, the mass of the upper platform has a more significant influence on the natural frequency and sensitivity of each component than that of the six legs, which indicates that the mass of the upper platform has a more significant influence on the stability and accuracy of the electrohydraulic platform than that of the six legs.

(2) When the position posture is in the positive position, the sensitivity of the six legs presents symmetry; that is, the sensitivity change curve of the two legs is the same. This is because the coupling between the legs is reduced when the position posture is in the symmetric position. When the posture was in an asymmetric position, the sensitivity of the six legs did not show symmetry.

\section{Data Availability}

The data used to support the findings of this study are included within the article. 


\section{Conflicts of Interest}

The authors declare that they have no conflicts of interest.

\section{Acknowledgments}

This work was supported in part by the National Key R\&D Project under grant 2019YFB2005303, in part by the National Natural Science Foundation of China (no. 51805468), by the Natural Science Foundation of Jiangsu Province under grant BK20180175, in part by the Key R\&D Program of Xuzhou of Jiangsu Province under grant KC18096, and in part by the Natural Science Foundation of Hebei Province under grant E2020203090.

\section{References}

[1] Y. Ting, C.-C. Li, and T. V. Nguyen, "Composite controller design for a 6DOF Stewart nanoscale platform," Precision Engineering, vol. 37, no. 3, pp. 671-683, 2013.

[2] L. Lyu, Z. Chen, and B. Yao, "Energy saving motion control of independent metering valves and pump combined hydraulic system," Mechatronics, IEEE/ASME Transactions on, vol. 24, no. 5, pp. 1909-1920, 2019.

[3] L. Lyu, Z. Chen, and B. Yao, "Development of pump and valves combined hydraulic system for both high tracking precision and high energy efficiency," IEEE Transactions on Industrial Electronics, vol. 66, no. 9, pp. 7189-7198, 2019.

[4] L. J. D. Plessis and J. A. Snyman, "An optimally re-configurable planar Gough-Stewart machining platform," Mechanism \& Machine Theory, vol. 41, no. 3, pp. 334-357, 2006.

[5] D. Kuehn, M. Schilling, T. Stark, M. Zenzes, and F. Kirchner, "System design and testing of the hominid robot charlie," Journal of Field Robotics, vol. 34, no. 4, pp. 666-703, 2017.

[6] K. Herbuś and P. Ociepka, "Integration of the virtual model of a Stewart platform with the avatar of a vehicle in a virtual reality," IOP Conference Series: Materials ENCE and Engineering, vol. 145, Article ID 042018, 2016.

[7] M. Alkhedher, U. Ali, and O. Mohamad, "Modeling, simulation and design of adaptive 6DOF vehicle stabilizer," in Proceedings of the 2019 8th International Conference on Modeling Simulation and Applied Optimization (ICMSAO), Manama, Bahrain, April 2019.

[8] M. Xia, Z. Xu, K. Han et al., "Dynamic disturbance force measurement platform for large moving device in spacecraft," Journal of Sound and Vibration, vol. 447, pp. 61-77, 2019.

[9] Y. Xu, H. Liao, L. Liu, and Y. Wang, "Modeling and robust $\mathrm{H}$-infinite control of a novel non-contact ultra-quiet Stewart spacecraft," Acta Astronautica, vol. 107, pp. 274-289, 2015.

[10] H. Yun, L. Liu, Q. Li, W. Li, and L. Tang, "Development of an isotropic Stewart platform for telescope secondary mirror," Mechanical Systems and Signal Processing, vol. 127, pp. 328344, 2019.

[11] Y. X. Su, B. Y. Duan, B. Peng, and R. D. Nan, "Singularity analysis of fine-tuning Stewart platform for large radio telescope using genetic algorithm," Mechatronics, vol. 13, no. 5, pp. 413-425, 2003.

[12] M. F. Shah, Z. Kausar, and M. M. Ahmad, "PID control and stability analysis of an ith leg of a six degree of freedom machining bed," Procedia Manufacturing, vol. 17, pp. 927934, 2018.

[13] A. S. Lafmejani, M. T. Masouleh, and A. Kalhor, "Trajectory tracking control of a pneumatically actuated 6-DOF
Gough-Stewart parallel robot using backstepping-sliding mode controller and geometry-based quasi forward kinematic method," Robotics and Computer-Integrated Manufacturing, vol. 54, pp. 96-114, 2018.

[14] L. Lyu, Z. Chen, and B. Yao, "Advanced valves and pump coordinated hydraulic control design to simultaneously achieve high accuracy and high efficiency," IEEE Transactions on Control Systems Technology, vol. 99, pp. 1-13, 2020.

[15] M. Wang, Y. Hu, Y. Sun et al., "An adjustable low-frequency vibration isolation Stewart platform based on electromagnetic negative stiffness," International Journal of Mechanical Sciences, vol. 181, Article ID 105714, 2020.

[16] Y.-S. Kim, H. Shi, N. Dagalakis, J. Marvel, and G. Cheok, "Design of a six-DOF motion tracking system based on a Stewart platform and ball-and-socket joints," Mechanism and Machine Theory, vol. 133, pp. 84-94, 2019.

[17] Y. Zhu, S. Tang, C. Wang, W. Jiang, X. Yuan, and Y. Lei, "Bifurcation characteristic research on the load vertical vibration of a hydraulic automatic gauge control system," Processes, vol. 7, no. 10, p. 718, 2019.

[18] S. Tang, S. Yuan, and Y. Zhu, "Deep learning-based intelligent fault diagnosis methods toward rotating machinery," IEEE Access, vol. 8, pp. 9335-9346, 2020.

[19] X. Yang, H. Wu, Y. Li, and B. Chen, "Dynamic isotropic design and decentralized active control of a six-axis vibration isolator via Stewart platform," Mechanism and Machine Theory, vol. 117, pp. 244-252, 2017.

[20] Z. Tong, C. Gosselin, and H. Jiang, "Dynamic decoupling analysis and experiment based on a class of modified GoughStewart parallel manipulators with line orthogonality," Mechanism and Machine Theory, vol. 143, Article ID 103636, 2020.

[21] F. Hu and X. Jing, "A 6-DOF passive vibration isolator based on Stewart structure with $X$-shaped legs," Nonlinear $D y$ namics, vol. 91, no. 1, pp. 157-185, 2018.

[22] Y. Zhu, S. Tang, L. Quan et al., "Extraction method for signal effective component based on extreme-point symmetric mode decomposition and Kullback-Leibler divergence," Journal of the Brazilian Society of Mechanical Sciences and Engineering, vol. 41, no. 2, 2019.

[23] Y. Zhu, P. F. Qian, S. N. Tang et al., "Amplitude-frequency characteristics analysis for vertical vibration of hydraulic AGC system under nonlinear action," AIP Advances, vol. 9, Article ID 035019, 2019.

[24] G. Ma, Y. Liu, Y. Sun et al., "Active vibration isolation for Stewart platform using backstepping and NFTSM control," in Proceedings of the Guidance, Navigation \& Control Conference, IEEE, Warsaw, Poland, April 2017.

[25] Y. Zhu, S. Tang, C. Wang et al., "Absolute stability condition derivation for position closed-loop system in hydraulic automatic gauge control," Processes, vol. 7, no. 10, p. 766, 2019.

[26] W. Li, E. Li, L. Ji et al., "Mechanism and propagation characteristics of rotating stall in a mixed-flow pump," Renewable Energy, vol. 153, pp. 74-92, 2020.

[27] X. Wang, X. Yue, H. Dai, and J. Yuan, "Vibration suppression for post-capture spacecraft via a novel bio-inspired Stewart isolation system," Acta Astronautica, vol. 168, pp. 1-22, 2020.

[28] Y. Cheng, G. Ren, and S. L. Dai, "The multi-body system modelling of the Gough-Stewart platform for vibration control," Journal of Sound \& Vibration, vol. 271, no. 3-5, pp. 599-614, 2004.

[29] B. Afzali-Far, A. Andersson, K. Nilsson, and P. Lidström, "Influence of strut inertia on the vibrations in initially 
symmetric Gough-Stewart platforms-an analytical study," Journal of Sound and Vibration, vol. 352, pp. 142-157, 2015.

[30] B. Ko, J.-W. Park, and D. W. Kim, "A study on iterative learning control for vibration of Stewart platform," International Journal of Control, Automation and Systems, vol. 15, no. 1, pp. 258-266, 2017.

[31] J. Jiao, Y. Wu, K. Yu et al., "Dynamic modeling and experimental analyses of Stewart platform with flexible hinges," Journal of Vibration and Control, vol. 25, no. 1, pp. 151-171, 2018.

[32] W. Khalil and S. Guegan, "Inverse and direct dynamic modeling of Gough-Stewart robots," Robotics IEEE Transactions on, vol. 20, no. 4, pp. 754-761, 2009.

[33] S. Zhibo, L. Jinhao, Y. Chunzhan et al., "A small range six-axis accelerometer designed with high sensitivity DCB elastic element," Sensors, vol. 16, no. 9, p. 1552, 2016.

[34] X. Yang, H. Wu, B. Chen, S. Kang, and S. Cheng, "Dynamic modeling and decoupled control of a flexible Stewart platform for vibration isolation," Journal of Sound and Vibration, vol. 439, pp. 398-412, 2019.

[35] Q.-T. Huang, Z.-M. Ye, and Z.-Z. Tong, "Dynamics modeling and analysis of a type of high frequency 6-DOF parallel platform," Advances in Intelligent Systems and Computing, vol. 363, pp. 469-482, 2015. 